Article

\title{
Computing Birational Polynomial Surface Parametrizations without Base Points
}

\author{
Sonia Pérez-Díaz *,+(D) and Juan Rafael Sendra + \\ Departamento de Física y Matemáticas, Universidad de Alcalá, E-28871 Madrid, Spain; rafael.sendra@uah.es \\ * Correspondence: sonia.perez@uah.es \\ t These authors contributed equally to this work.
}

Received: 17 November 2020; Accepted: 10 December 2020; Published: 14 December 2020

check for updates

\begin{abstract}
In this paper, we present an algorithm for reparametrizing birational surface parametrizations into birational polynomial surface parametrizations without base points, if they exist. For this purpose, we impose a transversality condition to the base points of the input parametrization.
\end{abstract}

Keywords: proper (i.e., birational) parametrization; polynomial parametrization; base point

\section{Introduction}

Algebraic surfaces are mainly studied from three different, but related, points of view, namely: pure theoretical, algorithmic and because of their applications. In this paper, we deal with some computational problems of algebraic surfaces taking into account the potential applicability.

In many different applications, as for instance in geometric design (see e.g., [1]) parametric representations of surfaces are more suitable than implicit representations. Among the different types of parametric representations, one may distinguish radical parametrizations (see [2]) and rational parametrizations (see e.g., [3]), the first being tuples of fractions of nested radical of bivariate polynomials, and the second being tuples of fractions of bivariate polynomials; in both cases the tuples are with generic Jacobian of rank 2. Other parametric representations by means of series can be introduced, but this is not within the scope of this paper. One may observe that the set of rational parametrizations is a subclass of the class of radical parametrizations. Indeed, in [4], one can find an algorithm to decide whether a radical parametrization can be transformed by means of a change of the parameters into a rational parametrization; in this case, we say that a reparametrization has been performed.

Now, we consider a third type of parametric representation of the surface, namely, the polynomial parametrization. That is, tuple of bivariate polynomials with generic Jacobian of rank 2. Clearly the class of polynomial parametrizations is a subclass of the class of the rational parametrizations, and the natural question of deciding whether a given rational parametrization can be reparametrized into a polynomial parametrization appears. This is, indeed, the problem we deal with in the paper. Unfortunately the inclusion of each of these classes into the next one is strict, and hence the corresponding reparametrizations are not always feasible. In some practical applications, the alternative is to use piecewise parametrizations with the desired property (see e.g., $[5,6]$ ).

Before commenting the details of our approach to the problem, let us look at some reasons why polynomial parametrizations may be more interesting than rational ones. In general, rational parametr izations are dominant over the surface (i.e., the Zariski closure of its image is the surface), but not necessarily surjective. This may introduce difficulties when applying the parametric representation to a problem, since the answer might be within the non-covered area of the surface. For the curve case, polynomial parametrizations are always surjective (see [7]). For the surface case, the result is not so direct but there are some interesting results for polynomial parametrizations to be surjective (see [8]) 
as well as subfamilies of polynomial parametrizations that are surjective (see [9]). Another issue that could be mention is the numerical instability when the values, substituted in the parameters of the parametrizations, get close to the poles of the rational functions; note that, in this case, the denominators define algebraic curves which points are all poles of the parametrization. One may also think on the advantages of providing a polynomial parametrization instead of a rational parametrization, when facing surface integrals. Let us mention a last example of motivation: the algebra-geometric technique for solving non autonomous ordinary differential equations (see $[10,11])$. In these cases, the differential equation is seen algebraically and hence representing a surface. Then, under the assumption that this surface is rational (resp. radical) the general rational (resp. radical) solution, if it exists, of the differential equation is determined from a rational parametrization of the surface. This process may be simplified if the associated algebraic parametrization admits a polynomial parametrization.

Next, let us introduce, and briefly comment on, the notion of base points of a rational parametrization. A base point of a given rational parametrization is a common solution of all numerators and denominators of the parametrization (see e.g., [12,13]). The presence of this type of points is a serious obstacle when approaching many theoretical, algorithmic or applied questions related to the surface represented by the parametrizations; examples of this phenomenon can be found in, e.g., [14-17]. In addition, it happens that rational surface may admit, both, birational parametrizations with empty base locus and with non-empty base locus. Moreover, the behavior of the base locus is not controlled, at least to our knowledge, by the existing parametrization algorithms or when the resulting parametrization appears as the consequence of the intersection of higher dimension varieties, or as the consequence of cissoid, conchoid, offsetting, or any other geometric design process applied to a surface parametrization (see e.g., [18-21]).

In this paper, we solve the problem, by means of reparametrizations, of computing a birational polynomial parametrization without base points of a rational surface, if it exists. For this purpose, we assume that we are given a birational parametrization of the surface that has the property of being transversal (this is a notion introduced in the paper, see Section 3 for the precise definition). Essentially, the idea of transversality is to assume that the multiplicity of the base points is minimal. Since, by definition (see Section 3) this multiplicity is introduced as a multiplicity of intersection of two algebraic curves, one indeed is requiring the transversality of the corresponding tangents. In this paper, we have not approached the problem of eliminating this hypothesis, and we leave it as future work in case it exists.

The general idea to solve the problem is as follows. We are given a birational parametrization $\mathcal{P}$ and let $\mathcal{Q}$ be the searched birational polynomial parametrization without base points; let us say, first of all, that throughout the paper we work projectively. Then, there exists a birational map, say $\mathcal{S}_{\mathcal{P}}$, that relates both parametrizations as $\mathcal{Q}=\mathcal{P} \circ \mathcal{S}_{\mathcal{P}}$. Then, taking into account that the base locus of $\mathcal{S}_{\mathcal{P}}$ and $\mathcal{P}$ are the same, that they coincide also in multiplicity, and applying some additional properties on base points stated in Section 3.1, we introduce a 2-dimensional linear system of curves, associated to an effective divisor generated by the base points of $\mathcal{P}$. Then, using the transversality we prove that every basis of the linear system, composed with a suitable birational transformation, provides a reparametrization of $\mathcal{P}$ that yields to a polynomial parametrization with empty base locus.

To give a better picture of these ideas, let us briefly illustrate them here by means of an example. We consider the projective surface $\mathscr{S}$ defined by the polynomial

$$
\begin{gathered}
-2 w^{2} y^{2}+2 w^{2} y z+2 w^{2} z^{2}-w x y^{2}-w x y z-w x z^{2}-8 w y^{3}+5 w y^{2} z+5 w y z^{2}-3 w z^{3}+x^{2} y^{2}-2 x y^{3} \\
+4 x y^{2} z-2 x y z^{2}+y^{4}-4 y^{3} z+6 y^{2} z^{2}-4 y z^{3}+z^{4}
\end{gathered}
$$

is rational and can be birationally parametrized as

$$
\begin{array}{r}
\mathcal{P}=\left(t_{2}^{2} t_{3}^{2}+t_{3} t_{1}^{3}+t_{3} t_{2}^{2} t_{1}-t_{1}^{4}-2 t_{1}^{2} t_{2}^{2}-t_{2}^{4}:-t_{3}\left(t_{1}+t_{2}\right)\left(t_{1}^{2}-t_{1} t_{3}+t_{2}^{2}-t_{2} t_{3}\right)\right. \\
\left.: t_{3}^{2}\left(t_{1}+2 t_{2}\right)\left(t_{1}+t_{2}\right):\left(t_{1}^{2}+t_{2}^{2}\right)^{2}\right)
\end{array}
$$


$\mathcal{P}$ provides the affine non-polynomial parametrization

$$
\left(\frac{-t_{1}^{4}-2 t_{1}^{2} t_{2}^{2}-t_{2}^{4}+t_{1}^{3}+t_{1} t_{2}^{2}+t_{2}^{2}}{\left(t_{1}^{2}+t_{2}^{2}\right)^{2}},-\frac{\left(t_{1}+t_{2}\right)\left(t_{1}^{2}+t_{2}^{2}-t_{1}-t_{2}\right)}{\left(t_{1}^{2}+t_{2}^{2}\right)^{2}}, \frac{\left(t_{1}+2 t_{2}\right)\left(t_{1}+t_{2}\right)}{\left(t_{1}^{2}+t_{2}^{2}\right)^{2}}\right) .
$$

On the other hand, $\mathscr{S}$ can also be parametrized as

$$
\mathcal{Q}=\left(t_{1}^{2}+t_{2} t_{3}-t_{1} t_{3}-t_{3}^{2}: t_{2}^{2}-t_{2} t_{3}: t_{2}^{2}+t_{2} t_{1}: t_{3}^{2}\right),
$$

that provides the affine polynomial parametrization

$$
\left(t_{1}^{2}+t_{2}-t_{1}-1, t_{2}^{2}-t_{2}, t_{2}^{2}+t_{2} t_{1}\right)
$$

The question is how to compute $\mathcal{Q}$ from $\mathcal{P}$. Since both parametrizations are birational, there exists a birational change of parameters $\mathcal{S}_{\mathcal{P}}$ such that $\mathcal{Q}=\mathcal{P} \circ \mathcal{S}_{\mathcal{P}}$. Furthermore, it holds that the base locus of $\mathcal{S}_{\mathcal{P}}$ and $\mathcal{P}$ are the same. So, the problem of finding $\mathcal{Q}$ is reduced to the problem of determining a birational map $\mathcal{S}_{\mathcal{P}}$ satisfying that the base locus of $\mathcal{S}_{\mathcal{P}}$ and $\mathcal{P}$ are the same. For this purpose, we introduce a 2-dimensional linear system of curves, associated to an effective divisor generated by the base points of $\mathcal{P}$ and, using the transversality, we prove that every basis of the linear system provides a polynomial parametrization of $\mathcal{P}$ with empty base locus.

The structure of the paper is as follows. In Section 2, we introduce the notation and we recall some definitions and properties on base points, essentially taken from [12]. In Section 3 we state some additional required properties on base points, we introduce the notion of transversality of a base locus, both for birational maps of the projective plane and for rational surface projective parametrizations. Moreover, we establish some fundamental properties that require the transversality. Section 4 is devoted to state the theoretical frame for solving the central problem treated in the paper. In Section 5 , we derive the algorithm that is illustrated by means of some examples. We finish the paper with a section on conclusions.

\section{Preliminary on Basic Points and Notation}

In this section, we briefly recall some of the notions related to base points and we introduce some notation; for further results on this topic we refer to [12]. We distinguish three subsections. In Section 2.1, the notation that will be used throughout the paper is introduced. The next subsection focuses on birational surface parametrizations, and the third subsection on birational maps of the projective plane.

\subsection{Notation}

Let, first of all, start fixing some notation. Throughout this paper, $\mathbb{K}$ is an algebraically closed field of characteristic zero. $\bar{x}=\left(x_{1}, \ldots, x_{4}\right), \bar{y}=\left(y_{1}, \ldots, y_{4}\right)$ and $\bar{t}=\left(t_{1}, t_{2}, t_{3}\right) . \mathbb{F}$ is the algebraic closure of $\mathbb{K}(\bar{x}, \bar{y})$. In addition, $\mathbb{P}^{k}(\mathbb{K})$ denotes the $k$-dimensional projective space, and $\mathscr{G}\left(\mathbb{P}^{k}(\mathbb{K})\right)$ is the set of all projective transformations of $\mathbb{P}^{k}(\mathbb{K})$.

Furthermore, for a rational map

$$
\begin{array}{ccc}
\mathcal{M}: & \mathbb{P}^{k_{1}}(\mathbb{K}) & \stackrel{\mathbb{P}^{k_{2}}(\mathbb{K})}{ } \\
\bar{h}=\left(h_{1}: \cdots: h_{k_{1}+1}\right) & \longmapsto & \left(m_{1}(\bar{h}): \cdots: m_{k_{2}+1}(\bar{h})\right),
\end{array}
$$

where the non-zero $m_{i}$ are homogenous polynomial in $\bar{h}$ of the same degree, we denote by $\operatorname{deg}(\mathcal{M})$ the degree $\operatorname{deg}_{\bar{h}}\left(m_{i}\right)$, for $m_{i}$ non-zero, and by $\operatorname{degMap}(\mathcal{M})$ the degree of the map $\mathcal{M}$; that is, the cardinality of the generic fiber of $\mathcal{M}$ (see e.g., [22]). 
For $L \in \mathscr{G}\left(\mathbb{P}^{k_{2}}(\mathbb{K})\right)$, and $M \in \mathscr{G}\left(\mathbb{P}^{k_{1}}(\mathbb{K})\right)$ we denote the left composition and the right composition, respectively, by

$$
{ }^{L} \mathcal{M}:=L \circ \mathcal{M}, \mathcal{M}^{M}:=\mathcal{M} \circ M .
$$

Let $f \in \mathbb{L}\left[t_{1}, t_{2}, t_{3}\right]$ be homogeneous and non-zero, where $\mathbb{L}$ is a field extension of $\mathbb{K}$. Then $\mathscr{C}(f)$ denotes the projective plane curve defined by $f$ over the algebraic closure of $\mathbb{L}$.

Let $\mathscr{C}(f), \mathscr{C}(g)$ be two curves in $\mathbb{P}^{2}(\mathbb{K})$. For $A \in \mathbb{P}^{2}(\mathbb{K})$, we represent by mult $_{A}(\mathscr{C}(f), \mathscr{C}(g))$ the multiplicity of intersection of $\mathscr{C}(f)$ and $\mathscr{C}(g)$ at $A$. In addition, we denote by mult $(A, \mathscr{C}(f))$ the multiplicity of $\mathscr{C}(f)$ at $A$.

Finally, $\mathscr{S} \subset \mathbb{P}^{3}(\mathbb{K})$ represents a rational projective surface.

\subsection{Case of Surface Parametrizations}

In this subsection, we consider a rational parametrization of the projective rational surface $\mathscr{S}$, namely,

$$
\begin{aligned}
& \mathcal{P}: \quad \mathbb{P}^{2}(\mathbb{K}) \quad \rightarrow \quad \mathscr{S} \subset \mathbb{P}^{3}(\mathbb{K}) \\
& \bar{t} \quad \longmapsto\left(p_{1}(\bar{t}): \cdots: p_{4}(\bar{t})\right),
\end{aligned}
$$

where $\bar{t}=\left(t_{1}, t_{2}, t_{3}\right)$ and the $p_{i}$ are homogenous polynomials of the same degree such that $\operatorname{gcd}\left(p_{1}, \ldots, p_{4}\right)=1$.

Definition 1. $A$ base point of $\mathcal{P}$ is an element $A \in \mathbb{P}^{2}(\mathbb{K})$ such that $p_{i}(A)=0$ for every $i \in\{1,2,3,4\}$. We denote by $\mathscr{B}(\mathcal{P})$ the set of base points of $\mathcal{P}$. That is $\mathscr{B}(\mathcal{P})=\mathscr{C}\left(p_{1}\right) \cap \cdots \cap \mathscr{C}\left(p_{4}\right)$.

In order to deal with the base points of the parametrization, we introduce the following auxiliary polynomials:

$$
\begin{aligned}
& W_{1}(\bar{x}, \bar{t}):=\sum_{i=1}^{4} x_{i} p_{i}\left(t_{1}, t_{2}, t_{3}\right) \\
& W_{2}(\bar{y}, \bar{t}):=\sum_{i=1}^{4} y_{i} p_{i}\left(t_{1}, t_{2}, t_{3}\right),
\end{aligned}
$$

where $x_{i}, y_{i}$ are new variables. We will work with the projective plane curves $\mathscr{C}\left(W_{i}\right)$ in $\mathbb{P}^{3}(\mathbb{F})$. Similarly, for $M=\left(M_{1}: M_{2}: M_{3}\right) \in \mathscr{G}\left(\mathbb{P}^{3}(\mathbb{K})\right)$, we define,

$$
\begin{aligned}
& W_{1}^{M}(\bar{x}, \bar{t}):=\sum_{i=1}^{4} x_{i} M_{i}(\mathcal{P}(\bar{t})) \\
& W_{2}^{M}(\bar{y}, \bar{t}):=\sum_{i=1}^{4} y_{i} M_{i}(\mathcal{P}(\bar{t})) .
\end{aligned}
$$

Remark 1. Sometimes, we will need to specify the parametrization in the polynomials above. In those cases, we will write $W_{i}^{\mathcal{P}}$ or $W_{i}^{M, \mathcal{P}}$ instead of $W_{i}$ or $W_{i}^{M}$; similarly, we may write $\mathscr{C}\left(W_{1}^{\mathcal{P}}\right)$ and $\mathscr{C}\left(W_{1}^{M, \mathcal{P}}\right)$.

Using the multiplicity of intersection of these two curves, we define the multiplicity of a base point as follows.

Definition 2. The multiplicity of a base point $A \in \mathscr{B}(\mathcal{P})$ is $\operatorname{mult}_{A}\left(\mathscr{C}\left(W_{1}\right), \mathscr{C}\left(W_{2}\right)\right)$, that is, is the multiplicity of intersection at $A$ of $\mathscr{C}\left(W_{1}\right)$ and $\mathscr{C}\left(W_{2}\right)$; we denote it by

$$
\operatorname{mult}(A, \mathscr{B}(\mathcal{P})):=\operatorname{mult}_{A}\left(\mathscr{C}\left(W_{1}\right), \mathscr{C}\left(W_{2}\right)\right)
$$

In addition, we define the multiplicity of the base points locus of $\mathcal{P}$, denoted $\operatorname{mult}(\mathscr{B}(\mathcal{P}))$, as

$$
\operatorname{mult}(\mathscr{B}(\mathcal{P})):=\sum_{A \in \mathscr{B}(\mathcal{P})} \operatorname{mult}(A, \mathscr{B}(\mathcal{P}))=\sum_{A \in \mathscr{B}(\mathcal{P})} \operatorname{mult}_{A}\left(\mathscr{C}\left(W_{1}\right), \mathscr{C}\left(W_{2}\right)\right)
$$

Note that, since $\operatorname{gcd}\left(p_{1}, \ldots, p_{4}\right)=1$, the set $\mathscr{B}(\mathcal{P})$ is either empty of finite. 
For the convenience of the reader we recall here some parts of Proposition 2 in [12].

Lemma 1. If $L \in \mathscr{G}\left(\mathbb{P}^{3}(\mathbb{K})\right)$, then:

1. If $A \in \mathscr{B}(\mathcal{P})$, then

$$
\operatorname{mult}\left(A, \mathscr{C}\left(W_{1}^{L}\right)\right)=\operatorname{mult}\left(A, \mathscr{C}\left(W_{2}^{L}\right)\right)=\min \left\{\operatorname{mult}\left(A, \mathscr{C}\left(p_{i}\right)\right) \mid i=1, \ldots, 4\right\} .
$$

2. If $A \in \mathscr{B}(\mathcal{P})$, then the tangents to $\mathscr{C}\left(W_{1}^{L}\right)$ at $A$ (similarly to $\mathscr{C}\left(W_{2}^{L}\right)$ ), with the corresponding multiplicities, are the factors in $\mathbb{K}[\bar{x}, \bar{t}] \backslash \mathbb{K}[\bar{x}]$ of

$$
\epsilon_{1} x_{1} T_{1}+\epsilon_{2} x_{2} T_{2}+\epsilon_{3} x_{3} T_{3}+\epsilon_{4} x_{4} T_{4}
$$

where $T_{i}$ is the product of the tangents, counted with multiplicities, of $\mathscr{C}\left(L_{i}(\mathcal{P})\right)$ at $A$, and where $\epsilon_{i}=1$ if $\operatorname{mult}\left(A, \mathscr{C}\left(L_{i}(\mathcal{P})\right)\right)=\min \left\{\operatorname{mult}\left(A, \mathscr{C}\left(L_{i}(\mathcal{P})\right)\right) \mid i=1, \ldots, 4\right\}$ and 0 otherwise.

2.3. Case of rational maps of $\mathbb{P}^{2}(\mathbb{K})$

In this subsection, let

$$
\begin{array}{lllc}
\mathcal{S}: & \mathbb{P}^{2}(\mathbb{K}) & -\rightarrow & \mathbb{P}^{2}(\mathbb{K}) \\
& \bar{t}=\left(t_{1}: t_{2}: t_{3}\right) & \longmapsto & \mathcal{S}(\bar{t})=\left(s_{1}(\bar{t}): s_{2}(\bar{t}): s_{3}(\bar{t})\right),
\end{array}
$$

where $\operatorname{gcd}\left(s_{1}, s_{2}, s_{3}\right)=1$, is a dominant rational transformation of $\mathbb{P}^{2}(\mathbb{K})$.

Definition 3. $A \in \mathbb{P}^{2}(\mathbb{K})$ is a base point of $\mathcal{S}(\bar{t})$ if $s_{1}(A)=s_{2}(A)=s_{3}(A)=0$. That is, the base points of $\mathcal{S}$ are the intersection points of the projective plane curves, $\mathscr{C}\left(s_{i}\right)$, defined over $\mathbb{K}$ by $s_{i}(\bar{t}), i=1,2,3$. We denote by $\mathscr{B}(\mathcal{S})$ the set of base points of $\mathcal{S}$.

We introduce the polynomials

$$
\begin{aligned}
& V_{1}=\sum_{i=1}^{3} x_{i} s_{i}(\bar{t}) \in \mathbb{K}(\bar{x}, \bar{y})[\bar{t}] \\
& V_{2}=\sum_{i=1}^{3} y_{i} s_{i}(\bar{t}) \in \mathbb{K}(\bar{x}, \bar{y})[\bar{t}],
\end{aligned}
$$

where $x_{i}, y_{j}$ are new variables and we consider the curves $\mathscr{C}\left(V_{i}\right)$ over the field $\mathbb{F}$; compare with (3). Similarly, for every $L \in \mathscr{G}\left(\mathbb{P}^{2}(\mathbb{K})\right)$ we introduce the polynomials

$$
\begin{aligned}
V_{1}^{L} & =\sum_{i=1}^{3} x_{i} L_{i}(\mathcal{S}) \in \mathbb{K}(\bar{x}, \bar{y})[\bar{t}] \\
V_{2}^{L} & =\sum_{i=1}^{3} y_{i} L_{i}(\mathcal{S}) \in \mathbb{K}(\bar{x}, \bar{y})[\bar{t}],
\end{aligned}
$$

Remark 2. Sometimes, we will need to specify the rational map in the polynomials above. In those cases, we will write $V_{i}^{\mathcal{S}}$ or $V_{i}^{L, \mathcal{S}}$ instead of $V_{i}$ or $V_{i}^{L}$; similarly, we may write $\mathscr{C}\left(V_{1}^{\mathcal{S}}\right)$ and $\mathscr{C}\left(V_{1}^{L, \mathcal{S}}\right)$.

As we did in Section 2.2, we have the following notion of multiplicity.

Definition 4. For $A \in \mathscr{B}(\mathcal{S})$, we define the multiplicity of intersection of $A$, and we denote it by $\operatorname{mult}(A, \mathscr{B}(\mathcal{S}))$, as

$$
\operatorname{mult}(A, \mathscr{B}(\mathcal{S})):=\operatorname{mult}_{A}\left(\mathscr{C}\left(V_{1}\right), \mathscr{C}\left(V_{2}\right)\right)
$$


In addition, we define the multiplicity of the base points locus of $\mathcal{S}$, denoted $\operatorname{mult}(\mathscr{B}(\mathcal{S})$ ), as (note that, since $\operatorname{gcd}\left(s_{1}, s_{2}, s_{3}\right)=1, \mathscr{B}(\mathcal{S})$ is either finite or empty)

$$
\operatorname{mult}(\mathscr{B}(\mathcal{S})):=\sum_{A \in \mathscr{B}(\mathcal{S})} \operatorname{mult}(A, \mathscr{B}(\mathcal{S}))=\sum_{A \in \mathscr{B}(\mathcal{S})} \operatorname{mult}_{A}\left(\mathscr{C}\left(V_{1}\right), \mathscr{C}\left(V_{2}\right)\right)
$$

The next result is a direct extension of Proposition 2 in [12] to the case of birational transformation of $\mathbb{P}^{2}(\mathbb{K})$.

Lemma 2. If $L \in \mathscr{G}\left(\mathbb{P}^{2}(\mathbb{K})\right)$ then

1. $\mathscr{B}(\mathcal{S})=\mathscr{C}\left(V_{1}^{L}\right) \cap \mathscr{C}\left(V_{2}^{L}\right) \cap \mathbb{P}^{2}(\mathbb{K})$.

2. Let $A \in \mathscr{B}(\mathcal{S})$ then

$$
\operatorname{mult}\left(A, \mathscr{C}\left(V_{1}^{L}\right)\right)=\operatorname{mult}\left(A, \mathscr{C}\left(V_{2}^{L}\right)\right)=\min \left\{\operatorname{mult}\left(A, \mathscr{C}\left(s_{i}\right)\right) \mid i=1,2,3\right\} .
$$

3. Let $A \in \mathscr{B}(\mathcal{S})$. The tangents to $\mathscr{C}\left(V_{1}^{L}\right)$ at $A$ (similarly to $\mathscr{C}\left(V_{2}^{L}\right)$ ), with the corresponding multiplicities, are the factors in $\mathbb{K}[\bar{x}, \bar{t}] \backslash \mathbb{K}[\bar{x}]$ of

$$
\epsilon_{1} x_{1} T_{1}+\epsilon_{2} x_{2} T_{2}+\epsilon_{3} x_{3} T_{3}
$$

where $T_{i}$ is the product of the tangents, counted with multiplicities, of $\mathscr{C}\left(L_{i}(\mathcal{S})\right)$ at $A$, and where $\epsilon_{i}=1$ if $\operatorname{mult}\left(A, \mathscr{C}\left(L_{i}(\mathcal{S})\right)\right)=\min \left\{\operatorname{mult}\left(A, \mathscr{C}\left(L_{i}(\mathcal{S})\right)\right) \mid i=1,2,3\right\}$ and 0 otherwise.

\section{Transversal Base Locus}

In this section, we present some new results on base points that complement those in [12] and we introduce and analyze the notion of transversality in conexion with the base locus.

Throughout this section, let $\mathcal{S}=\left(s_{1}: s_{2}: s_{3}\right)$, with $\operatorname{gcd}\left(s_{1}, s_{2}, s_{3}\right)=1$, be as in (6). In the sequel, we assume that $\mathcal{S}$ is birational. Let the inverse of $\mathcal{S}$ be denoted by $\mathcal{R}=\left(r_{1}: r_{2}: r_{3}\right)$; that is $\mathcal{R}:=\mathcal{S}^{-1}$. In addition, we consider a rational surface parametrization $\mathcal{P}=\left(p_{1}: \cdots: p_{4}\right)$, with $\operatorname{gcd}\left(p_{1}, \ldots, p_{4}\right)=1$, be as in (1). We assume that $\mathcal{P}$ is birational.

\subsection{Further Results on Base Points}

We start analyzing the rationality of the curve $\mathscr{C}\left(V_{i}^{L}\right)$ (see (8)).

Lemma 3. There exists a non-empty open subset $\Omega_{1}$ of $\mathscr{G}\left(\mathbb{P}^{2}(\mathbb{K})\right)$ such that if $L \in \Omega_{1}$ then $\mathscr{C}\left(V_{1}^{L}\right)$ is a rational curve. Furthermore,

$$
\mathcal{V}_{1}\left(\bar{x}, h_{1}, h_{2}\right)=\mathcal{R}^{L^{-1}}\left(h_{1} x_{3}, h_{2} x_{3},-\left(h_{1} x_{1}+x_{2} h_{2}\right)\right)
$$

is a birational parametrization of $\mathscr{C}\left(V_{1}^{L}\right)$.

Proof. We start proving that for every $L \in \mathscr{G}\left(\mathbb{P}^{2}(\mathbb{K})\right), V_{1}^{L}$ is irreducible. Indeed, let $L=\left(\sum \lambda_{i} t_{i}: \sum \mu_{i} t_{i}\right.$ : $\left.\sum \gamma_{i} t_{i}\right) \in \mathscr{G}\left(\mathbb{P}^{2}(\mathbb{K})\right)$. Then $V_{1}^{L}=\left(\lambda_{1} x_{1}+\mu_{1} x_{2}+\gamma_{1} x_{3}\right) s_{1}+\left(\lambda_{2} x_{1}+\mu_{2} x_{2}+\gamma_{2} x_{3}\right) s_{2}+\left(\lambda_{3} x_{1}+\mu_{3} x_{2}+\right.$ $\left.\gamma_{3} x_{3}\right) s_{3} \cdot \operatorname{gcd}\left(s_{1}, s_{2}, s_{3}\right)=1$ and $\operatorname{gcd}\left(\lambda_{1} x_{1}+\mu_{1} x_{2}+\gamma_{1} x_{3}, \lambda_{2} x_{1}+\mu_{2} x_{2}+\gamma_{2} x_{3}, \lambda_{3} x_{1}+\mu_{3} x_{2}+\gamma_{3} x_{3}\right)=1$ because the determinant of the matrix associated to $L$ is non-zero. Therefore, $V_{1}^{L}$ is irreducible.

In the following, to define the open set $\Omega_{1}$, let $\mathcal{L}\left(t_{1}, t_{2}, t_{3}\right)=\left(\mathcal{L}_{1}: \mathcal{L}_{2}: \mathcal{L}_{3}\right)$ be a generic element of $\mathscr{G}\left(\mathbb{P}^{2}(\mathbb{K})\right)$; that is, $\mathcal{L}_{i}=z_{i, 1} t_{1}+z_{i, 2} t_{2}+z_{i, 3} t_{3}$, where $z_{i, j}$ are undetermined coefficients satisfying that the determinant of the corresponding matrix is not zero. Furthermore, for $L \in \mathscr{G}\left(\mathbb{P}^{2}(\mathbb{K})\right.$ ), we denote by $\bar{z}^{L}$ the coefficient list of $L$. We also introduce the polynomial $R^{\mathcal{L}}=x_{1} \mathcal{L}^{1}+x_{2} \mathcal{L}^{2}+x_{3} \mathcal{L}^{3}=$ $\left(\sum z_{i, 1} x_{i}\right) t_{1}+\left(\sum z_{i, 2} x_{i}\right) t_{2}+\left(\sum z_{i, 3} x_{i}\right) t_{3}$. Similarly, for $L \in \mathscr{G}\left(\mathbb{P}^{2}(\mathbb{K})\right)$, we denote $R^{L}=R_{\mathcal{L}}\left(\bar{z}^{L}, \bar{x}, \bar{t}\right)$. 
We consider the birational extension $\mathcal{R}_{\bar{x}}: \mathbb{P}^{2}(\mathbb{F}) \rightarrow \mathbb{P}^{2}(\mathbb{F})$ of $\mathcal{R}$ from $\mathbb{P}^{2}(\mathbb{K})$ to $\mathbb{P}^{2}(\mathbb{F})$. Let $\mathcal{U}_{\bar{x}} \subset$ $\mathbb{P}^{2}(\mathbb{F})$ be the open set where the $\mathcal{R}_{\bar{x}}$ is bijective; say that $\mathcal{U}_{\bar{x}}=\mathbb{P}^{2}(\mathbb{F}) \backslash \Delta$. We express the close set $\Delta$ as $\Delta=\Delta_{1} \cup \Delta_{2}$ where $\Delta_{1}$ is either empty or it is a union of finitely many curves, and $\Delta_{2}$ is either empty or finite many points. We fix our attention in $\Delta_{1}$. Let $f(\bar{t})$ be the defining polynomial of $\Delta_{1}$. Let $Z\left(\bar{z}, t_{1}, t_{2}\right)$ be the remainder of $f$ when diving by $V_{1}^{\mathcal{L}}$ w.r.t $t_{3}$. Note that $R^{\mathcal{L}}$ does not divide $f$ since $R^{\mathcal{L}}$ is irreducible and depends on $\bar{z}$. Hence $Z$ is no zero. Let $\alpha(\bar{z})$ be the numerator of a non-zero coefficient of $Z$ w.r.t. $\left\{t_{1}, t_{2}\right\}$ and let $\beta(\bar{z})$ the l.c.m. of the denominators of all coefficients of $Z$ w.r.t. $\left\{t_{1}, t_{2}\right\}$. Then, we define $\Omega_{1}$ as

$$
\Omega_{1}=\left\{L \in \mathscr{G}\left(\mathbb{P}^{2}(\mathbb{K})\right) \mid \alpha\left(\bar{z}^{L}\right) \beta\left(\bar{z}^{L}\right) \neq 0\right\}
$$

We observe that, by construction, if $L \in \Omega_{1}$ then $\mathscr{C}\left(R^{L}\right) \cap \mathcal{U}_{\bar{x}}$ is dense in $\mathscr{C}\left(R^{L}\right)$.

Let $\bar{a}, \bar{b} \in \mathscr{C}\left(R^{L}\right) \cap \mathcal{U}_{\bar{x}}$ be two different points, then by injectivity $\mathcal{R}_{\bar{x}}\left(\mathscr{C}\left(R^{L}\right)\right)$ contains at least two points, namely $\mathcal{R}_{\bar{x}}(\bar{a})$ and $\mathcal{R}_{\bar{x}}(\bar{b})$. In this situation, since $\mathcal{R}_{\bar{x}}\left(\mathscr{C}\left(R^{L}\right)\right)$ and $\mathscr{C}\left(V_{1}\right)$ are irreducible we get that $\overline{\mathcal{R}_{\bar{x}}\left(\mathscr{C}\left(R^{L}\right)\right)}=\mathscr{C}\left(V_{1}\right)$, and hence $\mathscr{C}\left(V_{1}\right)$ is a rational curve $\mathbb{P}^{2}(\mathbb{F})$. Furthermore, one easily may check that $\mathcal{V}_{1}$ parametrizes $\mathscr{C}\left(V_{1}\right)$ and it is proper since $\mathcal{R}$ is birational.

Remark 3. Note that $\mathcal{V}_{1}\left(t_{3}, 0,-t_{1}, t_{1}, t_{2}\right)=\mathcal{R}^{L^{-1}}\left(-t_{1} t_{1},-t_{1} t_{2},-t_{1} t_{3}\right)=\mathcal{R}^{L^{-1}}(\bar{t})$. Hence

$$
{ }^{L} \mathcal{S}\left(\mathcal{V}_{1}\left(t_{3}, 0,-t_{1}, t_{1}, t_{2}\right)\right)={ }^{L} \mathcal{S}\left(\mathcal{R}^{L^{-1}}(\bar{t})\right)=\left(t_{1}: t_{2}: t_{3}\right) .
$$

Therefore,

$$
L_{i}\left(\mathcal{S}\left(\mathcal{V}_{1}\left(t_{3}, 0,-t_{1}, t_{1}, t_{2}\right)\right)=t_{i} \cdot \wp(\bar{t}), i=1,2,3\right.
$$

Next lemma analyzes the rationality of the curves $\mathscr{C}\left(L_{i}(\mathcal{S})\right)$ where $L=\left(L_{1}: L_{2}: L_{3}\right) \in \mathscr{G}\left(\mathbb{P}^{2}(\mathbb{K})\right)$.

Lemma 4. There exists a non-empty Zariski open subset $\Omega_{2}$ of $\mathscr{G}\left(\mathbb{P}^{2}(\mathbb{K})\right)$ such that if $L \in \mathscr{G}\left(\mathbb{P}^{2}(\mathbb{K})\right)$ then $\mathscr{C}\left(L_{i}(\mathcal{S})\right)$, where $i \in\{1,2,3\}$, is rational.

Proof. Let $\mathcal{U}$ be the open subset where $\mathcal{R}$ is a bijective map, and let $\left\{\rho_{j, 1} t_{1}+\rho_{j, 2} t_{2}+\rho_{j, 3} t_{3}\right\}_{j=1, \ldots, n}$ be the linear forms defining the lines, if any, included in $\mathbb{P}^{2}(\mathbb{K}) \backslash \mathcal{U}$. Then, we take $\Omega_{2}=\cap_{j=1}^{n} \Sigma_{j}$ where

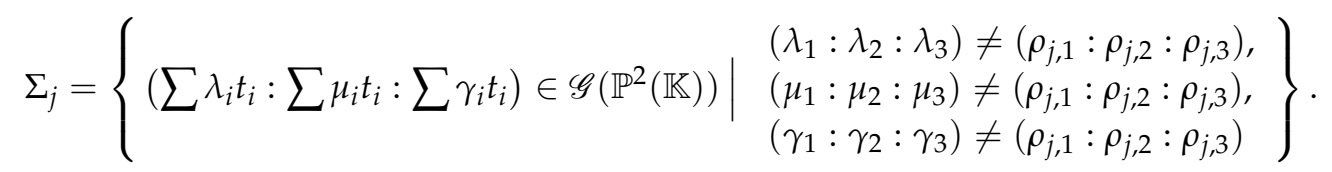

Now, let $L=\left(L_{1}: L_{2}: L_{3}\right) \in \Omega_{2}$. By construction, $\mathscr{C}\left(L_{i}\right) \cap \mathcal{U}$ is dense in $\mathscr{C}\left(L_{i}\right)$, for $i \in\{1,2,3\}$. In this situation, reasoning as in the last part of the proof of Lemma 3 , we get that $\overline{\mathcal{R}\left(\mathscr{C}\left(L_{i}\right)\right)}=$ $\mathscr{C}\left(L_{i}(\mathcal{S})\right)$. Therefore, $\mathscr{C}\left(L_{i}(\mathcal{S})\right)$ is rational.

The following lemma follows from Lemma 2.

Lemma 5. If $L \in \mathscr{G}\left(\mathbb{P}^{2}(\mathbb{K})\right)$ then

1. $\mathscr{B}(\mathcal{S})=\mathscr{B}\left({ }^{L} \mathcal{S}\right)$.

2. For $A \in \mathscr{B}(\mathcal{S})$ it holds that $\operatorname{mult}(A, \mathscr{B}(\mathcal{S}))=\operatorname{mult}\left(A, \mathscr{B}\left({ }^{L} \mathcal{S}\right)\right)$.

3. $\operatorname{mult}(\mathscr{B}(\mathcal{S}))=\operatorname{mult}\left(\mathscr{B}\left({ }^{L} \mathcal{S}\right)\right)$.

Proof. (1) Let $A \in \mathscr{B}(\mathcal{S})$ then $s_{1}(A)=s_{2}(A)=s_{3}(A)=0$. Thus, $L_{1}(\mathcal{S})(A)=L_{2}(\mathcal{S})(A)=$ $L_{3}(\mathcal{S})(A)=0$. So, $A \in \mathscr{B}\left({ }^{L} \mathcal{S}\right)$. Conversely, let $A \in \mathscr{B}\left({ }^{L} \mathcal{S}\right)$. Then expressing $L(\mathcal{S}(A))=\overline{0}$ in terms of matrices, since $L$ is invertible, we have that $\mathcal{S}(A)=L^{-1}(0,0,0)=(0,0,0)$. Thus, $A \in \mathscr{B}(\mathcal{S})$.

(2) and (3) follows from Theorem 5 in [12]. 
Lemma 6. There exists a non-empty Zariski open subset $\Omega_{3}$ of $\mathscr{G}\left(\mathbb{P}^{2}(\mathbb{K})\right)$ such that if $L \in \Omega_{3}$ then for every $A \in \mathscr{B}(\mathcal{S})$ it holds that

$$
\operatorname{mult}\left(A, \mathscr{C}\left(V_{1}^{L}\right)\right)=\operatorname{mult}\left(A, \mathscr{C}\left(L_{1}(\mathcal{S})\right)\right)=\operatorname{mult}\left(A, \mathscr{C}\left(L_{2}(\mathcal{S})\right)\right)=\operatorname{mult}\left(A, \mathscr{C}\left(L_{3}(\mathcal{S})\right)\right)
$$

Proof. Let $A \in \mathscr{B}(\mathcal{S})$. Then, by Lemma 2(2), we have that

$$
m_{A}:=\operatorname{mult}\left(A, \mathscr{C}\left(V_{1}^{L}\right)\right)=\min \left\{\operatorname{mult}\left(A, \mathscr{C}\left(s_{i}\right) \mid i \in\{1,2,3\}\right\}, \forall L \in \mathscr{G}\left(\mathbb{P}^{2}(\mathbb{K})\right) .\right.
$$

Let us assume w.l.o.g. that the minimum above is reached for $i=1$. Then all $\left(m_{A}-1\right)$-order derivatives of the forms $s_{i}$ vanish at $A$, and there exists an $m_{A}$-order partial derivative of $s_{1}$ not vanishing at $A$. Let us denote this partial derivative as $\partial^{m_{A}}$.

Now, let $\mathcal{L}$ be as in the proof of Lemma 3. Then,

$$
g_{i}(\bar{z}):=\partial^{m_{A}} \mathcal{L}_{i}(\mathcal{S})(A)=z_{i, 1} \partial^{m_{A}} S_{1}(A)+z_{i, 2} \partial^{m_{A}} S_{2}(A)+z_{i, 3} \partial^{m_{A}} S_{3}(A) \in \mathbb{K}[\bar{z}]
$$

is a non-zero polynomial because $\partial^{m_{A}} s_{1}(A) \neq 0$. We then consider the open subset (see proof of Lemma 3 for the notation $\bar{z}^{L}$ )

$$
\Omega_{A}=\left\{L \in \mathscr{G}\left(\mathbb{P}^{2}(\mathbb{K})\right) \mid g_{1}\left(\bar{z}^{L}\right) g_{2}\left(\bar{z}^{L}\right) g_{3}\left(\bar{z}^{L}\right) \neq 0\right\} \neq \varnothing .
$$

In this situation, we take

$$
\Omega_{3}=\bigcap_{A \in \mathscr{B}(\mathcal{S})} \Omega_{A}
$$

Note that, since $\mathscr{B}(\mathcal{S})$ is finite then $\Omega_{3}$ is open. Moreover, since $\mathscr{G}\left(\mathbb{P}^{2}(\mathbb{K})\right)$ is irreducible then $\Omega_{3}$ is not empty.

Let us prove that $\Omega_{3}$ satisfies the property in the statement of the lemma. Let $L \in \Omega_{3}$ and $A \in \mathscr{B}(\mathcal{S})$. Let $m_{A}$ be as in (11). Then all partial derivatives of $L_{i}(\mathcal{S})$, of any order smaller than $m_{A}$, vanishes at $A$. Moreover, since $L \in \Omega_{3} \subset \Omega_{A}$, it holds that $\partial^{m_{A}} L_{i}(\mathcal{S})(A) \neq 0$ for $i=1,2,3$. Therefore,

$$
\operatorname{mult}\left(A, \mathscr{C}\left(V_{1}^{L}\right)\right)=m_{A}=\operatorname{mult}\left(A, \mathscr{C}\left(L_{1}(\mathcal{S})\right)=\operatorname{mult}\left(A, \mathscr{C}\left(L_{2}(\mathcal{S})\right)=\operatorname{mult}\left(A, \mathscr{C}\left(L_{3}(\mathcal{S})\right)\right.\right.\right.
$$

Remark 4. We note that the proofs of Lemmas 5 and 6 are directly adaptable to the case of birational surface parametrizations. So, both lemmas hold if $M \in \mathscr{G}\left(\mathbb{P}^{3}(\mathbb{K})\right)$ and we replace $\mathcal{S}$ by the birational surface parametrizaion $\mathcal{P}$ and ${ }^{L} \mathcal{S}$ by ${ }^{M} \mathcal{P}:=M \circ \mathcal{P}$.

In the following, we denote by $\operatorname{Sing}(\mathcal{D})$, the set of singularities of an algebraic plane curve $\mathcal{D}$.

Corollary 1. Let $\Omega_{3}$ be the open subset in Lemma 6 and $L \in \Omega_{3}$. It holds that

1. $\cap_{i=1}^{3} \operatorname{Sing}\left(\mathscr{C}\left(L_{i}(\mathcal{S})\right)\right) \cap \mathscr{B}(\mathcal{S}) \subset \operatorname{Sing}\left(\mathscr{C}\left(V_{1}^{L}\right)\right)$.

2. Let $A \in \mathscr{B}(\mathcal{S})$. The tangents to $\mathscr{C}\left(V_{1}^{L}\right)$ at $A$, with the corresponding multiplicities, are the factors in $\mathbb{K}[\bar{x}, \bar{t}] \backslash \mathbb{K}[\bar{x}]$ of

$$
x_{1} T_{1}+x_{2} T_{2}+x_{3} T_{3}
$$

where $T_{i}$ is the product of the tangents, counted with multiplicities, to $\mathscr{C}\left(L_{i}(\mathcal{S})\right)$ at $A$.

3. Let $A \in \mathscr{B}(\mathcal{S})$, and let $T_{i}$ be the product of the tangents, counted with multiplicities, to $\mathscr{C}\left(L_{i}(\mathcal{S})\right)$, at $A$. If $\operatorname{gcd}\left(T_{1}, T_{2}, T_{3}\right)=1$, then

$$
\operatorname{mult}_{A}\left(\mathscr{C}\left(V_{1}^{L}\right), \mathscr{C}\left(V_{2}^{L}\right)\right)=\operatorname{mult}\left(A, \mathscr{C}\left(L_{i}(\mathcal{S})\right)^{2}, i \in\{1,2,3\}\right.
$$


Proof.

(1) $\quad$ Let $A \in \cap_{i=1}^{3} \operatorname{Sing}\left(\mathscr{C}\left(L_{i}(\mathcal{S})\right)\right) \cap \mathscr{B}(\mathcal{S})$. By Lemma 6, $m:=\operatorname{mult}\left(A, \mathscr{C}\left(L_{i}(\mathcal{S})\right)\right)>0$, for $i \in$ $\{1,2,3\}$, and $\operatorname{mult}\left(A, \mathscr{C}\left(V_{1}^{L}\right)\right)=m>0$. So, $A \in \operatorname{Sing}\left(\mathscr{C}\left(V_{1}^{L}\right)\right)$.

(2) follows from Lemmas 2 and 6.

(3) By (2) the tangents to $\mathscr{C}\left(V_{1}^{L}\right)$ and to $\mathscr{C}\left(V_{2}^{L}\right)$ at $A$ are $\mathcal{T}_{1}:=\sum x_{i} T_{i}$ and $\mathcal{T}_{2}:=\sum y_{i} T_{i}$, respectively. Since $\operatorname{gcd}\left(T_{1}, T_{2}, T_{3}\right)=1$, then $\mathcal{T}_{i}$ is primitive, and hence $\operatorname{gcd}\left(\mathcal{T}_{1}, \mathcal{T}_{2}\right)=1$. That is, $\mathscr{C}\left(V_{1}^{L}\right)$ and $\mathscr{C}\left(V_{2}^{L}\right)$ intersect transversally at $A$. From here, the results follows.

\subsection{Transversality}

We start introducing the notion of transversality for birational maps of $\mathbb{P}^{2}(\mathbb{K})$.

Definition 5. We say that $\mathcal{S}$ is transversal if either $\mathscr{B}(\mathcal{S})=\varnothing$ or for every $A \in \mathscr{B}(\mathcal{S})$ it holds that (see (7))

$$
\operatorname{mult}(A, \mathscr{B}(\mathcal{S}))=\operatorname{mult}\left(A, \mathscr{C}\left(V_{1}\right)\right)^{2}
$$

In this case, we also say that the base locus of $\mathcal{S}$ is transversal.

In the following lemma, we see that the transversality is invariant under left composition with elements in $\mathscr{G}\left(\mathbb{P}^{2}(\mathbb{K})\right)$.

Lemma 7. If $\mathcal{S}$ is transversal, then for every $L \in \mathscr{G}\left(\mathbb{P}^{2}(\mathbb{K})\right)$ it holds that ${ }^{L} \mathcal{S}$ is transversal.

Proof. By Lemma $5(1), \mathscr{B}(\mathcal{S})=\mathscr{B}\left({ }^{L} \mathcal{S}\right)$. So, if $\mathscr{B}(\mathcal{S})=\varnothing$, there is nothing to prove. Let $A \in \mathscr{B}(\mathcal{S}) \neq$ $\varnothing$, and let $L:=\left(L_{1}: L_{2}: L_{3}\right)$. Then

$$
\begin{array}{rlr}
\operatorname{mult}\left(A, \mathscr{B}\left({ }^{L} \mathcal{S}\right)\right) & =\operatorname{mult}(A, \mathscr{B}(\mathcal{S})) & (\text { see Lemma 5(2)) } \\
& =\operatorname{mult}\left(A, \mathscr{C}\left(V_{1}\right)\right)^{2} & (\mathcal{S} \text { is transversal) } \\
& =\operatorname{mult}\left(A, \mathscr{C}\left(V_{1}^{L} \mathcal{S}\right)\right)^{2} & (\text { see Lemma 2(2) and Remark 2) }
\end{array}
$$

Therefore, ${ }^{L} \mathcal{S}$ is transversal.

The next lemma characterizes the transversality by means of the tangents of $\mathscr{C}\left(s_{i}\right)$ at the base points. A direct generalization of this lemma to the case of surface parametrizations appears in Lemma 10, and will be used in Algorithm 1 for checking the transversality.

Lemma 8. The following statements are equivalent

1. $\mathcal{S}$ is transversal.

2. For every $A \in \mathscr{B}(\mathcal{S})$ it holds that $\operatorname{gcd}\left(T_{1}, T_{2}, T_{3}\right)=1$, where $T_{i}$ is the product of the tangents, counted with multiplicities, to $\mathscr{C}\left(s_{i}\right)$ at $A$.

Proof. If $\mathscr{B}(\mathcal{S})=\varnothing$, the result if trivial. Let $\mathscr{B}(\mathcal{S}) \neq \varnothing$. First of all, we observe that, because of Lemma 7, we may assume w.l.o.g. that Lemma 6 applies to $\mathcal{S}$. So, by Definition 5, $\mathcal{S}$ is transversal if and only if for every $A \in \mathscr{B}(\mathcal{S})$ it holds that

$$
\operatorname{mult}(A, \mathscr{B}(\mathcal{S}))=\operatorname{mult}\left(A, \mathscr{C}\left(V_{1}\right)\right)^{2},
$$

and, by Definition 2, if and only if

$$
\operatorname{mult}\left(A, \mathscr{C}\left(V_{1}\right)\right)^{2}=\operatorname{mult}_{A}\left(\mathscr{C}\left(V_{1}\right), \mathscr{C}\left(V_{2}\right)\right) .
$$


Furthermore, using Theorem 2.3.3 in [23], we have that

$$
\operatorname{mult}_{A}\left(\mathscr{C}\left(V_{1}\right), \mathscr{C}\left(V_{2}\right)\right)=\operatorname{mult}\left(A, \mathscr{C}\left(V_{1}\right)\right) \operatorname{mult}\left(A, \mathscr{C}\left(V_{2}\right)\right)
$$

if and only $V_{1}$ and $V_{2}$ intersect transversally at $A$ i.e., if the curves have no common tangents at $A$ which is equivalent to $\operatorname{gcd}\left(T_{1}, T_{2}, T_{3}\right)=1$. The proof finishes taking into account that, by Lemma 6 $\operatorname{mult}\left(A, \mathscr{C}\left(V_{1}\right)\right)=\operatorname{mult}\left(A, \mathscr{C}\left(V_{2}\right)\right)$.

In the last part of this section, we analyze the relationship of the transversality of a birational map of the projective plane and the transversality of a birational projective surface parametrization. For this purpose, first we introduce the notion of transversality for parametrizations.

Definition 6. Let $\mathcal{P}$ be a birational surface parametrization of $\mathbb{P}^{3}(\mathbb{K})$. We say that $\mathcal{P}$ is transversal if either $\mathscr{B}(\mathcal{P})=\varnothing$ or for every $A \in \mathscr{B}(\mathcal{P})$ it holds that (see (3))

$$
\operatorname{mult}(A, \mathscr{B}(\mathcal{P}))=\operatorname{mult}\left(A, \mathscr{C}\left(W_{1}\right)\right)^{2}
$$

In this case, we say that the base locus of $\mathcal{P}$ is transversal.

We start with some technical lemmas. The next lemma states that transversality does not change under projective transformations of the cartesian coordinates, i.e., under left composition. This has to be taken into account when extending the results of this paper to the case of non-transversality.

Lemma 9. If $\mathcal{P}$ is transversal, then for every $M \in \mathscr{G}\left(\mathbb{P}^{2}(\mathbb{K})\right)$ it holds that ${ }^{M \mathcal{P}}$ is transversal.

Proof. The proof is analogous to the proof of Lemma 7.

The next lemma provides a characterization of the transversality of a parametrization by means of the tangents that will be used in Algorithm 1. The proof of this lemma is a direct generalization of the proof of Lemma 8.

Lemma 10. The following statements are equivalent

1. $\mathcal{P}$ is transversal.

2. For every $A \in \mathscr{B}(\mathcal{P})$ it holds that $\operatorname{gcd}\left(T_{1}, \ldots, T_{4}\right)=1$, where $T_{i}$ is the product of the tangents, counted with multiplicities, to $\mathscr{C}\left(p_{i}\right)$ at $A$.

The following lemma focusses on the behavior of the base points of $\mathcal{P}$ when right composing with elements in $\mathscr{G}\left(\mathbb{P}^{2}(\mathbb{K})\right)$.

Lemma 11. Let $L \in \mathscr{G}\left(\mathbb{P}^{2}(\mathbb{K})\right)$. It holds that

1. $\mathscr{B}(\mathcal{P})=L\left(\mathscr{B}\left(\mathcal{P}^{L}\right)\right)$. Furthermore, $A \in \mathscr{B}(\mathcal{P})$ if and only $L^{-1}(A) \in \mathscr{B}\left(\mathcal{P}^{L}\right)$.

2. For $A \in \mathscr{B}(\mathcal{P}), \operatorname{mult}(A, \mathscr{B}(\mathcal{P}))=\operatorname{mult}\left(L^{-1}(A), \mathscr{B}\left(\mathcal{P}^{L}\right)\right)$

3. $\operatorname{mult}(\mathscr{B}(\mathcal{P}))=\operatorname{mult}\left(\mathscr{B}\left(\mathcal{P}^{L}\right)\right)$.

4. If $\mathcal{P}$ is transversal then $\mathcal{P}^{L}$ is also transversal.

Proof. (1) $A \in \mathscr{B}(\mathcal{P})$ iff $p_{i}(A)=0$ for $i \in\{1, \ldots, 4\}$ iff $p_{i}\left(L\left(L^{-1}(A)\right)\right)=0$ for $i \in\{1, \ldots, 4\}$ iff $L^{-1}(A) \in \mathscr{B}\left(\mathcal{P}^{L}\right)$ iff $A \in L\left(\mathscr{B}\left(\mathcal{P}^{L}\right)\right)$. So (1) follows.

We consider the curves $\mathscr{C}\left(W_{i}^{\mathcal{P}}\right)$ and $\mathscr{C}\left(W_{i}^{\mathcal{P}^{L}}\right)$ (see Remark 1$)$, and we note that $\mathscr{C}\left(W_{i}^{\mathcal{P}^{L}}\right)$ is the transformation of $\mathscr{C}\left(W_{i}\right)$ under the birational transformation $L^{-1}$ of $\mathbb{P}^{2}(\mathbb{K})$. Now, (2) and (3) follow from Definition 2, and (4) from Lemma 10. 
The next results analyze the base loci of birational surface parametrizations assuming that there exists one of them with empty base locus.

Lemma 12. Let $\mathcal{P}$ and $\mathcal{Q}$ be two birational projective parametrizations of the same surface $\mathscr{S}$ such that $\mathcal{Q}(\mathcal{S})=\mathcal{P}$ and $\mathscr{B}(\mathcal{Q})=\varnothing$. It holds that

1. $\mathscr{B}(\mathcal{S})=\mathscr{B}(\mathcal{P})$.

2. If $A \in \mathscr{B}(\mathcal{S})$ then $\operatorname{deg}(\mathscr{S}) \operatorname{mult}(A, \mathscr{B}(\mathcal{S}))=\operatorname{mult}(A, \mathscr{B}(\mathcal{P}))$.

Proof. Since $\mathcal{Q}=\mathcal{P}(\mathcal{S})$ and $\mathscr{B}(\mathcal{Q})=\varnothing$, by Theorem 11 in [12] we get that $\left.\mathscr{B}\left({ }^{L_{\mathcal{S}}}\right)=\mathscr{B}^{L_{\mathcal{P}}}\right)$ for $L_{\mathcal{S}}$ in a certain open subset of $\mathscr{G}\left(\mathbb{P}^{2}(\mathbb{K})\right)$ and $L_{\mathcal{P}}$ in a certain open subset of $\mathscr{G}\left(\mathbb{P}^{3}(\mathbb{K})\right)$. Now, using Lemma 5 , and Remark 4 one concludes the proof of statement (1). Statement (2) follows from Theorem 11 in [12], taking into account that $\mathcal{Q}$ is birational.

Lemma 13. Let $\mathcal{P}$ and $\mathcal{Q}$ be two birational projective parametrizations of the same surface $\mathscr{S}$ such that $\mathcal{Q}(\mathcal{S})=\mathcal{P}$ and $\mathscr{B}(\mathcal{Q})=\varnothing$. Then, for every $A \in \mathscr{B}(\mathcal{S})$ it holds that (see (3) and (7))

$$
\operatorname{mult}\left(A, \mathscr{C}\left(W_{1}\right)\right)=\operatorname{mult}\left(A, \mathscr{C}\left(V_{1}\right)\right) \operatorname{deg}(\mathcal{Q}) .
$$

Proof. Let $\mathcal{P}=\left(p_{1}: \cdots: p_{4}\right)$, and $\mathcal{Q}=\left(q_{1}: \cdots: q_{4}\right)$, where $\operatorname{gcd}\left(q_{1}, \ldots, q_{4}\right)=1$. We know that $p_{i}=q_{i}(\mathcal{S})$. Moreover, since $\mathscr{B}(\mathcal{Q})=\varnothing$, by Theorem 10 in [12], we have that $\operatorname{gcd}\left(p_{1}, \ldots, p_{4}\right)=1$.

We start observing that because of Lemma 12 one has that $\mathscr{B}(\mathcal{S})=\mathscr{B}(\mathcal{P})$. Now, let us consider $L \in \mathscr{G}\left(\mathbb{P}^{2}(\mathbb{K})\right)$ and $M \in \mathscr{G}\left(\mathbb{P}^{3}(\mathbb{K})\right)$. Let $\mathcal{Q}^{*}={ }^{M} Q^{L^{-1}}, \mathcal{S}^{*}={ }^{L} \mathcal{S}$ and $\mathcal{P}^{*}={ }^{M} \mathcal{P}$. Note that $\mathcal{Q}^{*}\left(\mathcal{S}^{*}\right)=\mathcal{P}^{*}$. Moreover, $\mathscr{B}\left(\mathcal{Q}^{*}\right)=\varnothing$. Indeed: if $A \in \mathbb{P}^{3}(\mathbb{K})$ then $B:=L^{-1}(A) \in \mathbb{P}^{3}(\mathbb{K})$ and, since $\mathscr{B}(\mathcal{Q})=\varnothing$, $C:=\mathcal{Q}(B) \in \mathbb{P}^{3}(\mathbb{K})$. Therefore $\mathcal{Q}^{*}(A)=M(B) \in \mathbb{P}^{3}(\mathbb{K})$ and, in consequence, $\mathscr{B}\left(\mathcal{Q}^{*}\right)=\varnothing$. Moreover, $\mathcal{Q}^{*}$ and $\mathcal{P}^{*}$ parametrize the same surface. Furthermore, by Lemma $7, \mathcal{S}^{*}$ is transversal. Thus, $\mathcal{S}^{*}, \mathcal{P}^{*}, \mathcal{Q}^{*}$ satisfy the hypotheses of the lemma. On the other hand, by Lemma 5 and Remark 4 , we have that $\mathscr{B}\left(\mathcal{S}^{*}\right)=\mathscr{B}(\mathcal{S})=\mathscr{B}(\mathcal{P})=\mathscr{B}\left(\mathcal{P}^{*}\right)$. Furthermore, by Lemmas 1 and 2 we have that $\operatorname{mult}\left(A, \mathscr{C}\left(V_{1}\right)\right)=\operatorname{mult}\left(A, \mathscr{C}\left(V_{1}^{L}\right)\right)$ and $\operatorname{mult}\left(A, \mathscr{C}\left(W_{1}\right)\right)=\operatorname{mult}\left(A, \mathscr{C}\left(W_{1}^{M}\right)\right)$. Therefore, by Lemma 6 and Remark 4, we can assume w.l.o.g. that for every $A \in \mathscr{B}(\mathcal{S})=\mathscr{B}(\mathcal{P})$ it holds that

$$
\begin{aligned}
& \operatorname{mult}\left(A, \mathscr{C}\left(V_{1}\right)\right)=\operatorname{mult}\left(A, \mathscr{C}\left(s_{i}\right)\right) \text { for } i \in\{1,2,3\} \\
& \operatorname{mult}\left(A, \mathscr{C}\left(W_{1}\right)\right)=\operatorname{mult}\left(A, \mathscr{C}\left(p_{i}\right)\right) \text { for } i \in\{1,2,3,4\}
\end{aligned}
$$

Now, let $A \in \mathscr{B}(\mathcal{P})$ and let $m:=\operatorname{mult}\left(A, \mathscr{C}\left(V_{1}\right)\right)$. We can assume w.l.o.g that $A=(0: 0: 1)$. Let $T_{i}$ denote the product of the tangents to $s_{i}$ at $A$. Additionally, let $\operatorname{deg}(\mathcal{S})=\mathfrak{s} \operatorname{deg}(\mathcal{P})=\mathfrak{p}$, and $\operatorname{deg}(\mathcal{Q})=\mathfrak{q}$ Then, by (12), we may write:

$$
s_{i}=T_{i} t_{3}^{\mathfrak{s}-m}+g_{m+1, i} t_{3}^{\mathfrak{s}-m-1}+\cdots+g_{\mathfrak{s}, i}
$$

where $g_{j, i}\left(t_{1}, t_{2}\right)$ are homogeneous forms of degree $j$. In addition, let $q_{i}$ be expressed as

$$
q_{i}(\bar{t})=F_{\mathfrak{q}, i}+F_{\mathfrak{q}-1, i} t_{3}+\cdots+F_{\ell_{i}, i} t_{3}^{\mathfrak{q}-\ell_{i}}
$$

where $F_{j, i}\left(t_{1}, t_{2}\right)$ are homogeneous forms of degree $j$. Then

$$
p_{i}(\bar{t})=F_{\mathfrak{q}, i}\left(s_{1}, s_{2}\right)+F_{\mathfrak{q}-1, i}\left(s_{1}, s_{2}\right) s_{3}+\cdots+F_{\ell_{i}, i}\left(s_{1}, s_{2}\right) s_{3}^{\mathfrak{q}-\ell_{i}} .
$$

Using this expression and (13) it can be expressed as

$$
\begin{gathered}
p_{i}(\bar{t})=\left(F_{\mathfrak{q}, i}\left(T_{1}, T_{2}\right)+F_{\mathfrak{q}-1, i}\left(T_{1}, T_{2}\right) T_{3}+\cdots+F_{\ell_{i}, i}\left(T_{1}, T_{2}\right) T_{3}^{\mathfrak{q}-\ell_{i}}\right) t_{3}^{\mathfrak{q}(\mathfrak{s}-m)} \\
+\left(\text { terms of degree in } t_{3} \text { strictly smaller than } \mathfrak{q}(\mathfrak{s}-m)\right) .
\end{gathered}
$$


Let

$$
H_{i}:=F_{\mathfrak{q}, i}\left(T_{1}, T_{2}\right)+F_{\mathfrak{q}-1, i}\left(T_{1}, T_{2}\right) T_{3}+\cdots+F_{\ell_{i}, i}\left(T_{1}, T_{2}\right) T_{3}^{\mathfrak{q}-\ell_{i}} .
$$

Now, let us prove that $H_{i}$ is not identically zero. We first observe that $H_{i}=q_{i}\left(T_{1}, T_{2}, T_{3}\right)$ for $i \in\{1,2,3,4\}$. We also note that if there exists $i \in\{1,2,3,4\}$ such that $H_{i}=0$, by (12), it must happen that for all $i \in\{1,2,3,4\}$ it holds that $H_{i}=0$. Let $H_{1}$ be zero. Then, $\mathcal{T}=\left(T_{1}: T_{2}: T_{3}\right) \notin \mathbb{P}^{2}(\mathbb{K})$, because otherwise $\mathcal{T} \in \mathscr{B}(\mathcal{Q})$ and $\mathscr{B}(\mathcal{Q})=\varnothing$. Therefore, $\mathcal{T}$ is a curve parametrization. Thus, if $H_{i}=0$, then $\mathcal{T}$ parametrizes a common component of the four curves $\mathscr{C}\left(p_{i}\right)$. However, this implies that $\operatorname{gcd}\left(q_{1}, q_{2}, q_{3}, q_{4}\right) \neq 1$ which is a contradiction.

Thus, by $(12), \operatorname{mult}\left(A, \mathscr{C}\left(p_{1}\right)\right)=\operatorname{mult}\left(A, \mathscr{C}\left(W_{i}\right)\right)=\mathfrak{q} m=\operatorname{deg}(\mathcal{Q}) \operatorname{mult}\left(A, \mathscr{C}\left(V_{i}\right)\right)$.

We finish this section stating the relationship between the transversality of $\mathcal{S}$ and $\mathcal{P}$ under the assumption that $\mathcal{P}\left(\mathcal{S}^{-1}\right)$ does not have base points.

Theorem 1. Let $\mathcal{P}$ and $\mathcal{Q}$ be two birational projective parametrizations of the same surface $\mathscr{S}$ such that $\mathcal{Q}(\mathcal{S})=\mathcal{P}$ and $\mathscr{B}(\mathcal{Q})=\varnothing$. Then, $\mathcal{S}$ is transversal if and only if $\mathcal{P}$ is transversal.

Proof. Let $A \in \mathscr{B}(\mathcal{S})=\mathscr{B}(\mathcal{P})$. First we note that from Lemma 13, and Corollary 5 in [12], it holds that

$$
\operatorname{mult}\left(A, \mathscr{C}\left(W_{1}\right)\right)^{2}=\operatorname{mult}\left(A, \mathscr{C}\left(V_{1}\right)\right)^{2} \operatorname{deg}(Q)^{2}=\operatorname{mult}\left(A, \mathscr{C}\left(V_{1}\right)\right)^{2} \operatorname{deg}(\mathscr{S})
$$

Using Corollary 9 in [12], we have that

$$
\operatorname{mult}(A, \mathscr{B}(\mathcal{P}))=\operatorname{deg}(\mathscr{S}) \operatorname{mult}(A, \mathscr{B}(\mathcal{S}))
$$

Therefore,

$$
\operatorname{mult}(A, \mathscr{B}(\mathcal{S})) \operatorname{mult}\left(A, \mathscr{C}\left(W_{1}\right)\right)^{2}=\operatorname{mult}(A, \mathscr{B}(\mathcal{P})) \operatorname{mult}\left(A, \mathscr{C}\left(V_{1}\right)\right)^{2} .
$$

Thus, $\mathcal{S}$ is transversal if and only if $\mathcal{P}$ is transversal.

\section{Proper Polynomial Reparametrization}

In this section, we deal with the central problem of the paper, namely, the determination, if they exist, of proper (i.e., birational) polynomial parametrizations of rational surfaces. For this purpose, we distinguish several subsections. In the first subsection, we fix the general assumptions and we propose our strategy. In the second subsection, we perform the theoretical analysis, and in the last subsection we prove the existence of a linear subspace, computable from the input data, and containing the solution to the problem.

We start recalling what we mean with a polynomial projective parametrization. We say that a projective parametrization is polynomial if its dehomogenization w.r.t. the fourth component, taking $t_{i}=1$ for some $i \in\{1,2,3\}$, is polynomial; note that the fourth component of a polynomial projective parametrization has to be a power of $t_{i}$ for some $i \in\{1,2,3\}$. Clearly, a similar reasoning is applicable w.r.t. other dehomogenizations. On the other hand, we say that a parametrization is almost polynomial if its fourth component is the power of a linear form.

The important fact is that a rational surface admits a birational polynomial parametrization if and only if it admits a birational almost polynomial parametrization. Furthermore, if we have an almost polynomial parametrization, and its fourth component is a power of the linear form $L_{3}^{*}(\bar{t})$, we may consider two additional linear forms $L_{1}^{*}, L_{2}^{*}$ such that $L^{*}=\left(L_{1}^{*}: L_{2}^{*}: L_{3}^{*}\right) \in \mathscr{G}\left(\mathbb{P}^{2}(\mathbb{K})\right)$ and then the composition of the almost polynomial parametrization with $\left(L^{*}\right)^{-1}$ is a polynomial parametrization of the same surface. 


\subsection{General Assumptions and Strategy}

In our analysis we have two main assumptions. We assume that the rational surface $\mathscr{S}$ admits a polynomial birational parametrization with empty base locus. Throughout the rest of the paper, let us fix one of these parametrizations and denote it by $\mathcal{Q}$; that is,

$$
\mathcal{Q}(\bar{t})=\left(q_{1}(\bar{t}): q_{2}(\bar{t}): q_{3}(\bar{t}): q_{4}(\bar{t})\right)
$$

with $q_{i}$ homogenous polynomials of the same degree such that $\operatorname{gcd}\left(q_{1}, \ldots, q_{4}\right)=1$, is a proper polynomial parametrization of $\mathscr{S}$ satisfying that $\mathscr{B}(\mathcal{Q})=\varnothing$. Note that, by Corollary 6 in [12], the degree of $\mathscr{S}$ is then the square of a natural number. Moreover, we introduce a second assumption. We assume that we are given a transversal birational parametrization of $\mathscr{S}$. Note that, because Lemmas 9 and 11 , this hypothesis is invariant under right and/or left projective transformations. Throughout the rest of the paper, let us fix $\mathcal{P}$ as a transversal proper parametrization of $\mathscr{S}$, and let $\mathcal{P}$ be expressed as in (1).

Our goal is to reach $\mathcal{Q}$, or more precisely an almost polynomial parametrization of $\mathscr{S}$, from $\mathcal{P}$. For this purpose, first we observe that, since both $\mathcal{P}$ and $\mathcal{Q}$ are birational, they are related by means of a birational map of $\mathbb{P}^{2}(\mathbb{K})$, say $\mathcal{S}_{\mathcal{P}}$. More precisely, $\mathcal{S}_{\mathcal{P}}:=\mathcal{Q}^{-1} \circ \mathcal{P}$. In the following, we represent $\mathcal{S}_{\mathcal{P}}$ as

$$
\mathcal{S}_{\mathcal{P}}(\bar{t})=\left(s_{1}(\bar{t}): s_{2}(\bar{t}): s_{3}(\bar{t})\right),
$$

where $\operatorname{gcd}\left(s_{1}, s_{2}, s_{3}\right)=1$. Note that, because of Theorem 1 , since $\mathcal{P}$ is transversal, then $\mathcal{S}_{\mathcal{P}}$ is transversal. In addition, let $\mathcal{R}_{\mathcal{P}}:=\mathcal{S}_{\mathcal{P}}^{-1}(\bar{t})=\mathcal{P}^{-1} \circ \mathcal{Q}$. In the sequel, we represent $\mathcal{R}_{\mathcal{P}}$ as

$$
\mathcal{R}_{\mathcal{P}}(\bar{t})=\left(r_{1}(\bar{t}): r_{2}(\bar{t}): r_{3}(\bar{t})\right)
$$

where $\operatorname{gcd}\left(r_{1}, r_{2}, r_{3}\right)=1$.

So, in order to derive $\mathcal{Q}$ from $\mathcal{P}$ it would be sufficient to determine $\mathcal{S}_{\mathcal{P}}$, and hence $\mathcal{R}_{\mathcal{P}}$, because $\mathcal{Q}=\mathcal{P}\left(\mathcal{R}_{\mathcal{P}}\right)$. Furthermore if, instead of determining $\mathcal{S}_{\mathcal{P}}$, we obtain ${ }^{L} \mathcal{S}_{\mathcal{P}}:=L \circ \mathcal{S}_{\mathcal{P}}$, for some $L \in \mathscr{G}\left(\mathbb{P}^{2}(\mathbb{K})\right)$, then instead of $\mathcal{Q}$ we get

$$
\mathcal{P}\left(\left({ }^{L} \mathcal{S}_{\mathcal{P}}\right)^{-1}\right)=\mathcal{P}\left(\mathcal{R}_{\mathcal{P}}^{L^{-1}}\right)=\mathcal{Q}\left(L^{-1}\right)=\mathcal{Q}^{L^{-1}}
$$

which is almost polynomial, and hence solves the problem. Taking into account this fact we make the following two considerations:

1. We can assume w.l.o.g. that $\mathscr{B}(\mathcal{P}) \neq \varnothing$. Indeed, if $\mathscr{B}(\mathcal{P})=\varnothing$, by Theorem 10 and Corollary 9 in [12], we get that $\mathscr{B}\left(\mathcal{S}_{\mathcal{P}}\right)=\varnothing$. Furthermore, by Corollary 7 in [12], we obtained that $\operatorname{deg}\left(\mathcal{S}_{\mathcal{P}}\right)=1$. Thus, using that $\mathcal{Q}$ is indeed polynomial, we get that the fourth component of $\mathcal{P}$ is the power of a linear form, and therefore the input parametrization $\mathcal{P}$ would be already almost polynomial, and hence the problem would be solved.

2. We can assume w.l.o.g. that $\mathcal{S}_{\mathcal{P}}$ satisfies whatever property reachable by means of a left composition with elements in $\mathscr{G}\left(\mathbb{P}^{2}(\mathbb{K})\right)$, as for instance those stated in Lemma 3, or Lemma 4 , or Lemma 6. In particular, by Lemma 7, the transversality is preserved. In other words, in the set $\mathscr{R}$ of all birational transformations of $\mathbb{P}^{2}(\mathbb{K})$, we consider the equivalence relation $\sim$, defined as $\mathcal{S} \sim \mathcal{S}^{*}$ if there exists $L \in \mathscr{G}\left(\mathbb{P}^{2}(\mathbb{K})\right)$ such that $L \circ \mathcal{S}=\mathcal{S}^{*}$, and we work with the equivalence classes in $\mathscr{R} / \sim$.

Therefore, our strategy will be to find a birational map $\mathcal{M}$ of $\mathbb{P}^{2}(\mathbb{K})$ such that $\mathcal{P}\left(\mathcal{M}^{-1}\right)$ is almost polynomial. For this purpose, we will see that it is enough to determine a dominant rational transformation $\mathcal{M}$ of $\mathbb{P}^{2}(\mathbb{K})$ (later, we will prove that such a transformation is indeed birational) such that

1. $\operatorname{deg}(\mathcal{M})=\operatorname{deg}\left(\mathcal{S}_{\mathcal{P}}\right)$.

2. $\mathscr{B}(\mathcal{M})=\mathscr{B}\left(\mathcal{S}_{\mathcal{P}}\right)$. 
3. $\forall A \in \mathscr{B}(\mathcal{M})$ it holds that $\operatorname{mult}(A, \mathscr{B}(\mathcal{M}))=\operatorname{mult}\left(A, \mathscr{B}\left(\mathcal{S}_{\mathcal{P}}\right)\right)$.

The difficulty is that both $\mathcal{M}$ and $\mathcal{S}_{\mathcal{P}}$ are unknown. Nevertheless, by Corollary 10 and Theorem 3 in [12], we have that

$$
\operatorname{deg}\left(\mathcal{S}_{\mathcal{P}}\right)=\frac{\operatorname{deg}(\mathcal{P})}{\sqrt{\operatorname{deg}(\mathscr{S})}} .
$$

Note that $\operatorname{deg}(\mathcal{P})$ is given and $\operatorname{deg}(\mathscr{S})$ can be determined by applying, for instance, the formulas in [24] (see also [25]). On the other hand, taking into account Lemma 12, we can achieve our goal by focusing on $\mathcal{P}$. More precisely, we reformulate the above conditions into the equivalent following conditions.

Conditions 1 . We say that a rational dominant map $\mathcal{M}$ of $\mathbb{P}^{2}(\mathbb{K})$ satisfies Conditions 1 if

1. $\operatorname{deg}(\mathcal{M})=\frac{\operatorname{deg}(\mathcal{P})}{\sqrt{\operatorname{deg}(\mathscr{S})}}$.

2. $\mathscr{B}(\mathcal{M})=\mathscr{B}(\mathcal{P})$,

3. $\operatorname{mult}(A, \mathscr{B}(\mathcal{M}))=\frac{\operatorname{mult}(A, \mathscr{B}(\mathcal{P}))}{\operatorname{deg}(\mathscr{S})}$ for all $A \in \mathscr{B}(\mathcal{P})$.

In the following subsections, we will see that rational dominant maps satisfying Conditions 1 provide an answer to the polynomiality problem.

\subsection{Theoretical Analysis}

We start this analysis with some technical lemmas. For this purpose, $\mathscr{S}, \mathcal{Q}, \mathcal{P}, \mathcal{S}_{\mathcal{P}}, \mathcal{R}_{\mathcal{P}}$ are as in the previous subsection. We recall that $\mathcal{Q}\left(\mathcal{S}_{\mathcal{P}}\right)=\mathcal{P}, \mathscr{B}(\mathcal{Q})=\varnothing, \mathcal{R}_{\mathcal{P}}=\mathcal{S}_{\mathcal{P}}^{-1}, \mathcal{P}$ is transversal, and hence $\mathcal{S}_{\mathcal{P}}$ is also transversal. Moreover, by Lemma 12, $\mathcal{S}_{\mathcal{P}}$ satisfies Conditions 1 . Furthermore, in the sequel, let

$$
\overline{\mathcal{S}}(\bar{t})=\left(\bar{s}_{1}(\bar{t}): \bar{s}_{2}(\bar{t}): \bar{s}_{3}(\bar{t})\right)
$$

with $\operatorname{gcd}\left(\bar{s}_{1}, \bar{s}_{2}, \bar{s}_{3}\right)=1$, be dominant rational map of $\mathbb{P}^{2}(\mathbb{K})$ satisfying Conditions 1 .

Lemma 14. Let $\mathcal{M}$ be a birational map of $\mathbb{P}^{2}(\mathbb{K})$. Then, $\operatorname{deg}(\mathcal{M})=\operatorname{deg}\left(\mathcal{M}^{-1}\right)$.

Proof. We use the notation introduced in Lemma 3. We take $L \in \mathscr{G}\left(\mathbb{P}^{3}(\mathbb{K})\right)$ such that

1. $\mathscr{C}\left(V_{1}^{L}\right)$ is rational (see (8) for the definition of $V_{1}^{L}$ constructed from $\mathcal{M}$, and Lemma 3 for the existence of $L)$.

2. $\operatorname{gcd}\left(\eta_{1}^{L}, \eta_{3}^{L}\right)=1$, where $\mathcal{M}^{-1}=\left(\eta_{1}: \eta_{2}: \eta_{3}\right)$.

In addition, we consider a projective transformation $N(\bar{t})$ in the parameters $\bar{t}$ such that $\operatorname{deg}_{\bar{t}}\left(V_{1}^{L}(\bar{x}, N(\bar{t}))=\operatorname{deg}_{t_{2}}\left(V_{1}^{L}(\bar{x}, N(\bar{t}))\right.\right.$ and $\operatorname{deg}_{\bar{t}}\left(\eta_{1}^{L}(N(\bar{t}))=\operatorname{deg}_{t_{2}}\left(\eta_{1}^{L}(N(\bar{t}))=\right.\right.$ $\operatorname{deg}_{t_{2}}\left(\eta_{3}^{L}(N(\bar{t}))\right.$. Then, it holds

$$
\begin{array}{rlr}
\operatorname{deg}(\mathcal{M}) & =\operatorname{deg}_{\bar{t}}\left(V_{1}^{L}(\bar{x}, \bar{t})\right) & \\
& =\operatorname{deg}_{\bar{t}}\left(V_{1}^{L}(\bar{x}, N(\bar{t}))\right. & (M \text { is a proj. transf.) } \\
& =\operatorname{deg}_{t_{2}}\left(V_{1}^{L}(\bar{x}, N(\bar{t}))\right. & \text { (see above) } \\
& =\operatorname{deg}_{t_{2}}\left(\eta_{1}^{L}\left(N\left(x_{1}, h_{1}, x_{3}\right)\right) / \eta_{3}^{L}\left(N\left(x_{1}, h_{1}, x_{3}\right)\right)\right. & \left.\quad{ }^{*}\right) \\
& =\operatorname{deg}_{t_{2}}\left(\eta_{1}^{L}\left(\left(N\left(x_{1}, h_{1}, x_{3}\right)\right)\right)\right. & \left(\operatorname{gcd}\left(\eta_{1}^{L}, \eta_{3}^{L}\right)=1\right) \\
& =\operatorname{deg}\left(\mathcal{M}^{-1}\right) &
\end{array}
$$

* See Theorem 4.21 in [26].

Our goal will be to compute birational transformations satisfying Conditions 1. Next lemma shows that the birationality will be derived from Conditions 1 , and hence we will not have to check it computationally. 
Lemma 15. Let $\mathcal{M}$ be a rational dominant map of $\mathbb{P}^{2}(\mathbb{K})$. If $\mathcal{M}$ satisfies Conditions 1 , then $\mathcal{M}$ is birational.

Proof. Since $\operatorname{deg}(\mathcal{M})=\operatorname{deg}\left(\mathcal{S}_{\mathcal{P}}\right)$, and $\operatorname{mult}(\mathscr{B}(\mathcal{M}))=\operatorname{mult}\left(\mathscr{B}\left(\mathcal{S}_{\mathcal{P}}\right)\right)$, by Theorem 7 (a) in [12], we have that $\operatorname{deg} \operatorname{Map}(\mathcal{M})=\operatorname{degMap}\left(\mathcal{S}_{\mathcal{P}}\right)$. So $\overline{\mathcal{S}}$ is birational.

Therefore, since we have assume above (see (19)) that $\overline{\mathcal{S}}$ satisfies Conditions $1, \overline{\mathcal{S}}$ is birational (see Lemma 15). Let

$$
\overline{\mathcal{R}}(\bar{t})=\overline{\mathcal{S}}^{-1}(\bar{t})=\left(\bar{r}_{1}(\bar{t}): \bar{r}_{2}(\bar{t}): \bar{r}_{3}(\bar{t})\right)
$$

be its inverse. Clearly, $\overline{\mathcal{S}}(\overline{\mathcal{R}})=\left(t_{1}: t_{2}: t_{3}\right)$, which implies that $\bar{s}_{i}(\overline{\mathcal{R}}(\bar{t}))=t_{i} \wp(\bar{t})$, for $i \in$ $\{1,2,3\}$, and where $\operatorname{deg}(\wp)=\operatorname{deg}(\overline{\mathcal{S}})^{2}-1$ (see Lemma 14) and hence $\operatorname{deg}(\wp)=\operatorname{mult}(\mathscr{B}(\overline{\mathcal{S}}))=$ $\operatorname{mult}\left(\mathscr{B}\left(\mathcal{S}_{\mathcal{P}}\right)\right)=\operatorname{mult}(\mathscr{B}(\mathcal{P}))$. In the next result we prove that $\wp$ is directly related to $\mathscr{B}(\overline{\mathcal{S}})$, and using that $\mathscr{B}(\overline{\mathcal{S}})=\mathscr{B}\left(\mathcal{S}_{\mathcal{P}}\right)$, we study the common factor appearing in the composition $\mathcal{S}(\overline{\mathcal{R}})$. We start with a technical lemma.

Lemma 16. Let $L \in \mathscr{G}\left(\mathbb{P}^{2}(\mathbb{K})\right)$. It holds that

1. $\mathscr{B}\left(\mathcal{S}_{\mathcal{P}}^{L}\right)=\mathscr{B}\left(\mathcal{P}^{L}\right)=\mathscr{B}\left(\overline{\mathcal{S}}^{L}\right)$.

2. If $A \in \mathscr{B}\left(\mathcal{S}_{\mathcal{P}}^{L}\right)$ then $\operatorname{mult}\left(A, \mathscr{B}\left(\mathcal{S}_{\mathcal{P}}^{L}\right)\right)=\operatorname{mult}\left(A, \mathcal{P}^{L}\right) / \operatorname{deg}(\mathscr{S})$

3. $\overline{\mathcal{S}}^{L}$ satisfies Conditions 1 .

4. $\mathcal{S}_{\mathcal{P}}^{L}$ is transversal

5. If $\overline{\mathcal{S}}$ is transversal, then $\overline{\mathcal{S}}^{L}$ is transversal.

Proof.

(1) $\quad A \in \mathscr{B}\left(\mathcal{S}_{\mathcal{P}}^{L}\right)$ iff $\mathcal{S}_{\mathcal{P}}^{L}(A)=\overline{0}$ iff $\mathcal{S}_{\mathcal{P}}(L(A))=\overline{0}$ iff $L(A) \in \mathscr{B}\left(\mathcal{S}_{\mathcal{P}}\right)=\mathscr{B}(\mathcal{P})$ iff $\mathcal{P}^{L}(A)=$ $\mathcal{P}(L(A))=\overline{0}$ iff $A \in \mathscr{B}\left(\mathcal{P}^{L}\right)$. Moreover, the second equality follows as in the previous reasoning, taking into account that $\bar{S}$ satisfies Condition 1 , and hence $\mathscr{B}\left(\mathcal{S}_{\mathcal{P}}\right)=\mathscr{B}(\overline{\mathcal{S}})=\mathscr{B}(\mathcal{P})$.

(2) follows taking into account that the multiplicity of a point on a curve, as well as the multiplicity of intersection, does not change under projective transformations.

(3) Condition (1) follows taking into account that $\operatorname{deg}(L)=1$. Statement (1) implies condition (2). For condition (3), we apply statement (2) and that $\operatorname{mult}\left(A, \mathscr{B}\left(\overline{\mathcal{S}}^{L}\right)\right)=\operatorname{mult}(A, \mathscr{B}(\mathcal{S}))$ because the multiplicity of intersection does not change with $L$.

(4) and (5) follow arguing as in the proof of Lemma 11(4).

Theorem 2. Let $\overline{\mathcal{S}}$ be transversal. If $i \in\{1,2,3\}$ then $\bar{s}_{i}(\overline{\mathcal{R}})=t_{i} \wp(\bar{t})$ where $\operatorname{deg}(\wp(\bar{t}))=\operatorname{mult}(\mathscr{B}(\mathcal{P}))$ and such that $\wp$ is uniquely determined by $\mathscr{B}(\mathcal{P})$.

Proof. We first observe that we can assume w.l.o.g. that no base point of $\mathcal{P}$ is on the line at infinity $x_{3}=$ 0 . Indeed, let $L \in \mathscr{G}\left(\mathbb{P}^{2}(\mathbb{K})\right)$ be such that $\mathscr{B}(\mathcal{P})$ is contained in the affine plane $x_{3}=1$. We consider $\overline{\mathcal{S}}^{*}:=\overline{\mathcal{S}}^{L}=\left(\bar{s}_{1}^{*}: \bar{s}_{2}^{*}: \bar{s}_{3}^{*}\right)$ and $\overline{\mathcal{R}}^{*}:=\left(\overline{\mathcal{S}}^{L}\right)^{-1}$, then $\bar{s}_{i}^{*}\left(\overline{\mathcal{R}}^{*}\right)=\bar{s}_{i}^{*}\left(L^{-1}(\overline{\mathcal{R}})\right)$, and $\bar{s}_{i}^{*}=\bar{s}_{i}(L)$; hence $\bar{s}_{i}^{*}\left(\overline{\mathcal{R}}^{*}\right)=\bar{s}_{i}(\overline{\mathcal{R}})$. In addition, because of Lemma $16, \overline{\mathcal{S}}^{*}$ satisfies the hypothesis of the theorem.

Let $\mathscr{C}\left(\bar{V}_{1}\right)$ denote the curve associated to $\overline{\mathcal{S}}$ as in (7). By Lemma 3, taking $L$ in the corresponding open subset of $\mathscr{G}\left(\mathbb{P}^{2}(\mathbb{K})\right)$, we have that $\mathscr{C}\left(\bar{V}_{1}^{L}\right)$ is a rational curve. So, we assume w.l.o.g. that $\mathscr{C}\left(\bar{V}_{1}\right)$ is rational. Let $\overline{\mathcal{V}}\left(\bar{x}, h_{1}, h_{2}\right)$ be the rational parametrization of $\mathscr{C}\left(\bar{V}_{1}\right)$ provided by Lemma 3. We apply a Möbius transformation $\phi \in \mathscr{G}\left(\mathbb{P}^{1}(\mathbb{K})\right)$ such that if $\overline{\mathcal{W}}\left(\bar{x}, h_{1}, h_{2}\right)=\left(\bar{w}_{1}\left(\bar{x}, h_{1}, h_{2}\right)\right.$ : $\left.\bar{w}_{2}\left(\bar{x}, h_{1}, h_{2}\right): \bar{w}_{3}\left(\bar{x}, h_{1}, h_{2}\right)\right):=\overline{\mathcal{V}}\left(\bar{x}, \phi\left(h_{1}, h_{2}\right)\right)$ then the affine parametrization $\bar{\rho}\left(\bar{x}, h_{1}\right):=$ $\left(\bar{w}_{1}\left(\bar{x}, h_{1}, 1\right) / \bar{w}_{3}\left(\bar{x}, h_{1}, 1\right), \bar{w}_{2}\left(\bar{x}, h_{1}, 1\right) / \bar{w}_{3}\left(\bar{x}, h_{1}, 1\right)\right)$ is affinely surjective (see $\left.[7,23]\right)$. 
Now, let $A=\left(a_{1}: a_{2}: 1\right) \in \mathscr{B}(\mathcal{P})$. By Lemma $2, P \in \mathscr{C}\left(\bar{V}_{1}\right)$. We observe that, by taking $L$ in the open subset of Lemma 6, we may assume that

$$
m_{A}:=\operatorname{mult}\left(A, \mathscr{C}\left(\bar{V}_{1}\right)\right)=\operatorname{mult}\left(A, \mathscr{C}\left(\bar{s}_{i}\right)\right), i \in\{1,2,3\} .
$$

We consider the polynomial

$$
g_{A}=\operatorname{gcd}\left(\bar{w}_{1}\left(\bar{x}, h_{1}, h_{2}\right)-a_{1} \bar{w}_{3}\left(\bar{x}, h_{1}, h_{2}\right), \bar{w}_{2}\left(\bar{x}, h_{1}, h_{2}\right)-a_{2} \bar{w}_{3}\left(\bar{x}, h_{1}, h_{2}\right)\right) .
$$

Since the affine parametrization has been taken surjective, we have that

$$
\operatorname{deg}_{\bar{h}}\left(g_{A}\right)=m_{A}
$$

and that for every root $t_{0}$ of $g_{A}$ it holds that $\bar{\rho}\left(t_{0}\right)=\left(a_{1}, a_{2}\right)$. We write $\bar{w}_{i}$ as

$$
\bar{w}_{i}=g_{A} \cdot \bar{w}_{i}^{*}+a_{i} \bar{w}_{3}, i=1,2 .
$$

On the other hand, we express $\bar{s}_{i}$ as

$$
\bar{s}_{i}(\bar{t})=\bar{T}_{i, m_{A}}(\bar{t}) t_{3}^{\operatorname{deg}(\overline{\mathcal{S}})-m_{A}}+\cdots+\bar{T}_{i, \operatorname{deg}(\overline{\mathcal{S}})}(\bar{t}),
$$

where $\operatorname{deg}\left(\bar{T}_{i, j}\right)=j, j \in\left\{m_{A}, \ldots, \operatorname{deg}(\overline{\mathcal{S}})\right\}$, and $\bar{T}_{i, j}(\bar{t})=\sum_{k_{1}+k_{2}=j}\left(t_{1}-a_{1} t_{3}\right)^{k_{1}}\left(t_{2}-a_{2} t_{3}\right)^{k_{2}}$. Therefore

$$
\bar{s}_{i}(\overline{\mathcal{W}})=g_{A}^{m_{A}} \cdot\left(\bar{T}_{i, m_{A}}\left(\bar{w}_{1}^{*}, \bar{w}_{2}^{*}\right) \bar{w}_{3}^{\operatorname{deg}(\overline{\mathcal{S}})-m_{A}}+\cdots+g_{A}^{\operatorname{deg}(\overline{\mathcal{S}})-m_{A}} \bar{T}_{i, \operatorname{deg}(\overline{\mathcal{S}})}\left(\bar{w}_{1}^{*}, \bar{w}_{2}^{*}\right)\right) .
$$

In other words, $g_{A}$ divides $\bar{s}_{i}(\overline{\mathcal{W}})$. Now, for $B=\left(b_{1}: b_{2}: 1\right) \in \mathscr{B}(\mathcal{P})$, with $A \neq B$, it holds that $\operatorname{gcd}\left(g_{A}, g_{B}\right)=1$, since otherwise there would exist a root $t_{0}$ of $\operatorname{gcd}\left(g_{A}, g_{B}\right)$, and this implies that $\bar{\rho}\left(t_{0}\right)=\left(a_{1}, a_{2}\right)=\left(b_{1}, b_{2}\right)=\bar{\rho}\left(t_{0}\right)$ which is a contradiction. Therefore, we have that

$$
\bar{s}_{i}(\overline{\mathcal{W}})=\prod_{A \in \mathscr{B}(\mathcal{P})} g_{A}\left(\bar{x}, h_{1}, h_{2}\right)^{m_{A}} f_{i}\left(\bar{x}, h_{1}, h_{2}\right)
$$

We observe that the factor defined by the base points does not depend on $i$. Thus, since $\bar{s}_{i}(\overline{\mathcal{W}})$ does depend on $i$, we get that $f_{i}$ is the factor depending on $i$. Furthermore,

$$
\begin{array}{rlr}
\operatorname{deg}_{\bar{h}}\left(\prod_{\left.A \in \mathscr{B}(\mathcal{P}) g_{A}^{m_{A}}\right)}=\sum_{A \in \mathscr{B}(\mathcal{P})} \operatorname{deg}_{\bar{h}}\left(g_{A}\right)^{m_{A}}\right. & \\
& =\sum_{A \in \mathscr{B}(\mathcal{P})} m_{A}^{2} & \text { (see (22)) } \\
& =\sum_{A \in \mathscr{B}(\mathcal{P})} \operatorname{mult}\left(A, \mathscr{C}\left(\bar{V}_{1}\right)\right)^{2} & \text { (see (21)) } \\
& =\sum_{A \in \mathscr{B}(\mathcal{P})} \operatorname{mult}(A, \mathscr{B}(\overline{\mathcal{S}})) & (\overline{\mathcal{S}} \text { is transversal) } \\
& =\sum_{A \in \mathscr{B}(\mathcal{P})} \operatorname{mult}(A, \mathscr{B}(\overline{\mathcal{P}})) & \text { (See Conditions 1) } \\
& =\operatorname{mult}(\mathscr{B}(\overline{\mathcal{P}})) & \text { (See Definition 2) }
\end{array}
$$

Moreover, by Theorem 4.21 in [23], since $\overline{\mathcal{W}}$ is birational it holds that $\operatorname{deg}(\overline{\mathcal{W}})=\operatorname{deg}\left(\mathscr{C}\left(\bar{V}_{1}\right)\right)=$ $\operatorname{deg}(\overline{\mathcal{S}})$. Hence, $\operatorname{deg}\left(\overline{s_{i}}(\overline{\mathcal{W}})\right)=\operatorname{deg}(\overline{\mathcal{S}})^{2}=\operatorname{mult}(\mathscr{B}(\mathcal{P}))+1$. Therefore, $f_{i}$ in $(28)$ is a linear form.

In this situation, let us introduce the notation $\bar{t}^{*}:=\left(t_{3}, 0,-t_{1}, t_{1}, t_{2}\right)$ and $\bar{t}^{* *}=$ $\left(t_{3}, 0,-t_{1}, \phi^{-1}\left(t_{1}, t_{2}\right)\right)$. Then, for $i \in\{1,2,3\}$, we have that

$$
\begin{aligned}
& t_{i \wp}=\bar{s}_{i}(\overline{\mathcal{R}}) \\
& =\bar{s}_{i}\left(\overline{\mathcal{V}}\left(\bar{t}^{*}\right)\right) \\
& =\bar{s}_{i}\left(\overline{\mathcal{W}}\left(\bar{t}^{* *}\right)\right) \\
& =\prod_{A \in \mathscr{B}(\mathcal{P})} g_{A}\left(\bar{t}^{* *}\right)^{m_{A}} f_{i}\left(\bar{t}^{* *}\right)
\end{aligned}
$$


Taking into account that $\prod_{A \in \mathscr{B}(\mathcal{P})} g_{A}\left(\bar{t}^{* *}\right)^{m_{A}}$ does not depend on $i$, we get that $t_{1} f_{2}\left(\bar{t}^{* *}\right)=$ $t_{2} f_{1}\left(\bar{t}^{* *}\right)$. This implies that $t_{1}$ divides $f_{1}\left(\bar{t}^{* *}\right)$, and since $f_{1}\left(\bar{t}^{* *}\right)$ is linear we get that $t_{1}=\lambda f_{1}\left(\bar{t}^{* *}\right)$ for $\lambda \in \mathbb{K} \backslash\{0\}$. Then, substituting above, we get $\lambda f_{1}\left(\bar{t}^{* *}\right) f_{2}\left(\bar{t}^{* *}\right)=t_{2} f_{1}\left(\bar{t}^{* *}\right)$, which implies that $t_{2}=\lambda f_{2}\left(\bar{t}^{* *}\right)$. Similarly, for $t_{3}=\lambda f_{3}\left(\bar{t}^{* *}\right)$. Therefore, we get that

$$
\bar{s}_{i}(\overline{\mathcal{R}})=t_{i} \lambda \prod_{A \in \mathscr{B}(\mathcal{P})} g_{A}\left(\bar{t}^{* *}\right)^{m_{A}}, \text { with } \lambda \in \mathbb{K} \backslash\{0\}
$$

This concludes the proof.

For the next theorem, we recall that $\mathcal{S}_{\mathcal{P}}=\left(s_{1}: s_{2}: s_{3}\right)$ with $\operatorname{gcd}\left(s_{1}, s_{2}, s_{3}\right)=1$; see (17).

Theorem 3. Let $\overline{\mathcal{S}}$ be transversal. If $i \in\{1,2,3\}$ then $s_{i}(\overline{\mathcal{R}})=Z_{i}(\bar{t}) \wp(\bar{t})$ where $Z_{i}$ is a linear form, $\operatorname{deg}(\wp(\bar{t}))=\operatorname{mult}(\mathscr{B}(\mathcal{P}))$ and such that $\wp$ is uniquely determined by $\mathscr{B}(\mathcal{P})$.

Proof. We first observe that we can assume w.l.o.g. that no base point of $\mathcal{P}$ is on the line at infinity $x_{3}=$ 0 . Indeed, let $L \in \mathscr{G}\left(\mathbb{P}^{2}(\mathbb{K})\right)$ be such that $\mathscr{B}(\mathcal{P})$ is contained in the affine plane $x_{3}=1$. We consider $\mathcal{S}^{*}:=\mathcal{S}_{\mathcal{P}}^{L}=\left(s_{1}^{*}: s_{2}^{*}: s_{3}^{*}\right), \overline{\mathcal{S}}^{*}:=\overline{\mathcal{S}}^{L}$, and $\overline{\mathcal{R}}^{*}:=\left(\overline{\mathcal{S}}^{*}\right)^{-1}$. Then $s_{i}^{*}\left(\overline{\mathcal{R}}^{*}\right)=s_{i}\left(L\left(L^{-1}(\overline{\mathcal{R}})\right)\right)=s_{i}(\overline{\mathcal{R}})$. In addition, because of Lemma $16, \overline{\mathcal{S}}^{*}$ and $\mathcal{S}^{*}$ satisfy the hypotheses of the theorem.

Let $\mathscr{C}\left(\bar{V}_{1}\right), \overline{\mathcal{V}}\left(\bar{x}, h_{1}, h_{2}\right), \overline{\mathcal{W}}\left(\bar{x}, h_{1}, h_{2}\right)=\left(\bar{w}_{1}: \bar{w}_{2}: \bar{w}_{3}\right)$ and $\bar{\rho}$ be as in the proof of Theorem 2.

Now, let $A=\left(a_{1}: a_{2}: 1\right) \in \mathscr{B}(\mathcal{P})$. By Lemma $2, P \in \mathscr{C}\left(\bar{V}_{1}\right)$. We recall that $\mathscr{B}(\mathcal{P})=$ $\mathscr{B}(\overline{\mathcal{S}})=\mathscr{B}\left(\mathcal{S}_{\mathcal{P}}\right)$. Let $\Omega_{3}^{\overline{\mathcal{S}}}$ and $\Omega_{3}^{\mathcal{S}_{\mathcal{P}}}$ be the open subset in Lemma 6 applied to $\overline{\mathcal{S}}$ and $\mathcal{S}_{\mathcal{P}}$, respectively. Taking $L \in \Omega_{3}^{\overline{\mathcal{S}}} \cap \Omega_{3}^{\mathcal{S}_{\mathcal{P}}}$ (note that $\mathscr{G}\left(\mathbb{P}^{2}(\mathbb{K})\right.$ ) is irreducible and hence the previous intersection is non-empty), we may assume that

$$
m_{A}:=\operatorname{mult}\left(A, \mathscr{C}\left(\bar{V}_{1}\right)\right)=\operatorname{mult}\left(A, \mathscr{C}\left(\bar{s}_{i}\right)\right), i \in\{1,2,3\} .
$$

and

$$
\operatorname{mult}\left(A, \mathscr{C}\left(V_{1}\right)\right)=\operatorname{mult}\left(A, \mathscr{C}\left(s_{i}\right)\right), i \in\{1,2,3\} .
$$

Since $\mathcal{S}_{\mathcal{P}}$ and $\overline{\mathcal{S}}$ are transversal, and taking into account Condition 1, it holds that

$$
\operatorname{mult}\left(A, \mathscr{C}\left(V_{1}\right)\right)^{2}=\operatorname{mult}\left(A, \mathscr{B}\left(\mathcal{S}_{\mathcal{P}}\right)\right)=\operatorname{mult}(A, \mathscr{B}(\overline{\mathcal{S}}))=\operatorname{mult}\left(A, \mathscr{C}\left(\bar{V}_{1}\right)\right)^{2}
$$

Therefore,

$$
\operatorname{mult}\left(A, \mathscr{C}\left(V_{1}\right)\right)=m_{A}=\operatorname{mult}\left(A, \mathscr{C}\left(\bar{V}_{1}\right)\right) .
$$

We consider the polynomial $g_{A}=\operatorname{gcd}\left(\bar{w}_{1}-a_{1} \bar{w}_{3}, \bar{w}_{2}-a_{2} \bar{w}_{3}\right)$. Reasoning as in the Proof of Theorem 2 we get that

$$
\operatorname{deg}_{\bar{h}}\left(g_{A}\right)=m_{A}
$$

and that for every root $t_{0}$ of $g_{A}$ it holds that $\bar{\rho}\left(t_{0}\right)=\left(a_{1}, a_{2}\right)$. We write $\bar{w}_{i}$ as $\bar{w}_{i}=g_{A} \cdot \bar{w}_{i}^{*}+a_{i} \bar{w}_{3}$ for $i=\in\{1,2\}$.

On the other hand, by (25) and (26), we have that $\operatorname{mult}\left(A, \mathscr{C}\left(s_{i}\right)\right)=m_{A}$. Therefore, we can express $s_{i}$ as

$$
s_{i}(\bar{t})=T_{i, m_{A}}(\bar{t}) t_{3}^{\operatorname{deg}\left(\mathcal{S}_{\mathcal{P}}\right)-m_{A}}+\cdots+T_{i, \operatorname{deg}\left(\mathcal{S}_{\mathcal{P}}\right)}(\bar{t}),
$$

where $\operatorname{deg}\left(T_{i, j}\right)=j, j \in\left\{m_{A}, \ldots, \operatorname{deg}\left(\mathcal{S}_{\mathcal{P}}\right)\right\}$, and $T_{i, j}(\bar{t})=\sum_{k_{1}+k_{2}=j}\left(t_{1}-a_{1} t_{3}\right)^{k_{1}}\left(t_{2}-\right.$ $\left.a_{2} t_{3}\right)^{k_{2}}$. Therefore

$$
s_{i}(\overline{\mathcal{W}})=g_{A}^{m_{A}} \cdot\left(T_{i, m_{A}}\left(\bar{w}_{1}^{*}, \bar{w}_{2}^{*}\right) \bar{w}_{3}^{\operatorname{deg}\left(\mathcal{S}_{\mathcal{P}}\right)-m_{A}}+\cdots+g_{A}^{\operatorname{deg}\left(\mathcal{S}_{\mathcal{P}}\right)-m_{A}} T_{i, \operatorname{deg}(\mathcal{S})}\left(\bar{w}_{1}^{*}, \bar{w}_{2}^{*}\right)\right) .
$$


In other words, $g_{A}$ divides $s_{i}(\overline{\mathcal{W}})$. Now, for $B=\left(b_{1}: b_{2}: 1\right) \in \mathscr{B}(\mathcal{P})$, with $A \neq B$, reasoning as in the proof of Theorem 2, it holds that $\operatorname{gcd}\left(g_{A}, g_{B}\right)=1$. Therefore, we have that

$$
s_{i}(\overline{\mathcal{W}})=\prod_{A \in \mathscr{B}(\mathcal{P})} g_{A}\left(\bar{x}, h_{1}, h_{2}\right)^{m_{A}} f_{i}\left(\bar{x}, h_{1}, h_{2}\right)
$$

Furthermore,

$$
\begin{array}{rlr}
\operatorname{deg}_{\bar{h}}\left(\prod_{\left.A \in \mathscr{B}(\mathcal{P}) g_{A}^{m_{A}}\right)}=\sum_{A \in \mathscr{B}(\mathcal{P})} \operatorname{deg}_{\bar{h}}\left(g_{A}\right)^{m_{A}}\right. & \\
& =\sum_{A \in \mathscr{B}(\mathcal{P})} m_{A}^{2} & \text { (see (27)) } \\
& =\sum_{A \in \mathscr{B}(\mathcal{P})} \operatorname{mult}\left(A, \mathscr{C}\left(\bar{V}_{1}\right)\right)^{2} & \text { (see (24)) } \\
& =\sum_{A \in \mathscr{B}(\mathcal{P})} \operatorname{mult}(A, \mathscr{B}(\overline{\mathcal{S}})) & (\overline{\mathcal{S}} \text { is transversal) } \\
& =\sum_{A \in \mathscr{B}(\mathcal{P})} \operatorname{mult}(A, \mathscr{B}(\overline{\mathcal{P}})) & \text { (See Conditions 1) } \\
& =\operatorname{mult}(\mathscr{B}(\overline{\mathcal{P}})) & \text { (See Definition 2) }
\end{array}
$$

Moreover, by Theorem 4.21 in [23], since $\overline{\mathcal{W}}$ is birational it holds that $\operatorname{deg}(\overline{\mathcal{W}})=\operatorname{deg}\left(\mathscr{C}\left(\bar{V}_{1}\right)\right)=$ $\operatorname{deg}(\overline{\mathcal{S}})$. Hence, by Condition $1, \operatorname{deg}\left(s_{i}(\overline{\mathcal{W}})\right)=\operatorname{deg}\left(\mathcal{S}_{\mathcal{P}}\right) \operatorname{deg}(\overline{\mathcal{S}})=\operatorname{deg}\left(\mathcal{S}_{\mathcal{P}}\right)^{2}=\operatorname{mult}(\mathscr{B}(\mathcal{P}))+1$. Therefore, $f_{i}$ in (28) is a linear form.

In this situation, let us introduce the notation $\bar{t}^{*}:=\left(t_{3}, 0,-t_{1}, t_{1}, t_{2}\right)$ and $\bar{t}^{* *}=$ $\left(t_{3}, 0,-t_{1}, \phi^{-1}\left(t_{1}, t_{2}\right)\right)$. Then, for $i \in\{1,2,3\}$, we have that

$$
\begin{aligned}
& s_{i}(\overline{\mathcal{R}})=s_{i}\left(\overline{\mathcal{V}}\left(\bar{t}^{*}\right)\right) \\
& =s_{i}\left(\overline{\mathcal{W}}\left(\bar{t}^{* *}\right)\right) \\
& =\prod_{A \in \mathscr{B}(\mathcal{P})} g_{A}\left(\bar{t}^{* *}\right)^{m_{A}} f_{i}\left(\bar{t}^{* *}\right)
\end{aligned}
$$

This concludes the proof.

Corollary 2. If $\overline{\mathcal{S}}$ is transversal, there exists $L \in \mathscr{G}\left(\mathbb{P}^{2}(\mathbb{K})\right)$ such that $\overline{\mathcal{S}}={ }^{L} \mathcal{S}_{\mathcal{P}}$.

Proof. From Theorem 3, we get that $\mathcal{S}_{\mathcal{P}}(\overline{\mathcal{R}})=\left(Z_{1}(\bar{t}): Z_{2}(\bar{t}): Z_{3}(\bar{t})\right)$, where $Z_{i}$ is a linear form. Thus, ${ }^{L} \mathcal{S}_{\mathcal{P}}=\overline{\mathcal{S}}$, where $L \in \mathscr{G}\left(\mathbb{P}^{2}(\mathbb{K})\right)$ is the inverse of $\left(Z_{1}, Z_{2}, Z_{3}\right)$.

Corollary 3. The following statements are equivalent

1. $\overline{\mathcal{S}}$ is transversal.

2. There exists $L \in \mathscr{G}\left(\mathbb{P}^{2}(\mathbb{K})\right)$ such that $\overline{\mathcal{S}}={ }^{L} \mathcal{S}_{\mathcal{P}}$.

Proof. If (1) holds, then (2) follows from Corollary 2. Conversely, if (2) holds, then (1) follows from Lemma 7

\subsection{The Solution Space}

In this subsection we introduce a linear projective variety containing the solution to our problem and we show how to compute it. We start identifying the set of all projective curves, including multiple component curves, of a fixed degree $d$, with the projective space (see $[23,27,28]$ for further details)

$$
\mathscr{V}_{d}:=\mathbb{P}^{\frac{d(d+3)}{2}}(\mathbb{K}) .
$$

More precisely, we identify the projective curves of degree $d$ with the forms in $\mathbb{K}[\bar{t}]$ of degree $d$, up to multiplication by non-zero $\mathbb{K}$-elements. Now, these forms are identified with the elements in $\mathscr{V}_{d}$ corresponding to their coefficients, after fixing an order of the monomials. By abuse of notation, we will refer to the elements in $\mathscr{V}_{d}$ by either their tuple of coefficients, or the associated form, or the corresponding curve. 
Let $\mathcal{M}=\left(m_{1}(\bar{t}): m_{2}(\bar{t}): m_{3}(\bar{t})\right), \operatorname{gcd}\left(m_{1}, m_{2}, m_{3}\right)=1$, be a birational transformation of $\mathbb{P}^{2}(\mathbb{K})$. We consider $\mathscr{V}_{\operatorname{deg}(\mathcal{M})}$. Then, $m_{1}, m_{2}, m_{3} \in \mathscr{V}_{\operatorname{deg}(\mathcal{M})}$. Moreover, in $\mathscr{V}_{\operatorname{deg}(\mathcal{M})}$, we introduce the projective linear subspace

$$
\mathcal{L}(\mathcal{M}):=\left\{a_{1} m_{1}(\bar{t})+a_{2} m_{2}(\bar{t})+a_{3} m_{3}(\bar{t}) \mid\left(a_{1}: a_{2}: a_{3}\right) \in \mathbb{P}^{2}(\mathbb{K})\right\} .
$$

We observe that if $\left\{m_{1}, m_{2}, m_{3}\right\}$ are linearly dependent, then the image of $\mathbb{P}^{2}(\mathbb{K})$ via $\mathcal{M}^{-1}$ would be a line in $\mathbb{P}^{2}(\mathbb{K})$ which is impossible because $\mathcal{M}$ is birational on $\mathbb{P}^{2}(\mathbb{K})$. Therefore, the following holds.

Lemma 17. If $\mathcal{M}$ is a birational transformation of $\mathbb{P}^{2}(\mathbb{K})$, then $\operatorname{dim}(\mathcal{L}(\mathcal{M}))=2$.

Similarly, one has the next lemma.

Lemma 18. If $\mathcal{M}$ is a birational transformation of $\mathbb{P}^{2}(\mathbb{K})$ and $L \in \mathscr{G}\left(\mathbb{P}^{2}(\mathbb{K})\right)$ then $\mathcal{L}(\mathcal{M})=\mathcal{L}\left({ }^{L} \mathcal{M}\right)$.

Furthermore, the following theorem holds

Theorem 4. Let $\mathcal{M}$ be a birational transformation of $\mathbb{P}^{2}(\mathbb{K})$ and let $\left\{n_{1}, n_{2}, n_{3}\right\}$ be a basis of $\mathcal{L}(\mathcal{M})$ and $\mathcal{N}:=\left(n_{1}: n_{2}: n_{3}\right)$. There exists $L \in \mathscr{G}\left(\mathbb{P}^{2}(\mathbb{K})\right)$ such that ${ }^{L} \mathcal{M}=\mathcal{N}$.

Proof. Let $\mathcal{M}=\left(m_{1}: m_{2}: m_{3}\right)$, with $\operatorname{gcd}\left(m_{1}, m_{2}, m_{3}\right)=1$. Since $m_{1}, m_{2}, m_{3} \in \mathcal{L}(\mathcal{M})$, and $\left\{n_{1}, n_{2}, n_{3}\right\}$ is a basis of $\mathcal{L}(\mathcal{M})$, there exist $\left(\lambda_{i, 1}: \lambda_{i, 2}: \lambda_{i, 3}\right) \in \mathbb{P}^{2}(\mathbb{K})$ such that

$$
m_{i}=\sum \lambda_{i, j} n_{j}
$$

Since $\left\{m_{1}, m_{2}, m_{3}\right\}$ is also a basis of $\mathcal{L}(\mathcal{M})$, one has that $L:=\left(\sum \lambda_{1, j} t_{j}: \sum \lambda_{2, j} t_{j}: \sum \lambda_{3, j} t_{j}\right) \in$ $\mathscr{G}\left(\mathbb{P}^{2}(\mathbb{K})\right)$ and $\mathcal{N}=L \circ \mathcal{M}$.

Remark 5. Observe that, by Theorem 4, all bases of $\mathcal{L}(\mathcal{M})$ generate birational maps of $\mathbb{P}^{2}(\mathbb{K})$.

Corollary 4. Let $\mathcal{M}$ be a birational transformation of $\mathbb{P}^{2}(\mathbb{K})$. The following statements are equivalent

1. $\mathcal{M}$ is transversal.

2. There exists a basis $\left\{n_{1}, n_{2}, n_{3}\right\}$ of $\mathcal{L}(\mathcal{M})$ such that $\left(n_{1}: n_{2}: n_{3}\right)$ is transversal.

3. For all bases $\left\{n_{1}, n_{2}, n_{3}\right\}$ of $\mathcal{L}(\mathcal{M})$ it holds that $\left(n_{1}: n_{2}: n_{3}\right)$ is transversal.

Proof. It follows from Theorem 4 and Lemma 7.

In the following results we analyze the bases of $\mathcal{L}\left(\mathcal{S}_{\mathcal{P}}\right)$. So, $\mathcal{S}, \mathcal{R}, \mathcal{P}, \mathcal{Q}$ and $\bar{S}$ are as the in previous subsections.

Corollary 5. Let $\left\{m_{1}, m_{2}, m_{3}\right\}$ a basis of $\mathcal{L}\left(\mathcal{S}_{\mathcal{P}}\right)$. Then, $\left(m_{1}: m_{2}: m_{3}\right)$ satisfies Conditions 1.

Proof. It is a direct consequence of Theorem 4 .

Corollary 6. If $\mathcal{M}:=\left(m_{1}: m_{2}: m_{3}\right)$ is transversal and satisfies Condition 1 , then $\left\{m_{1}, m_{2}, m_{3}\right\}$ is a basis of $\mathcal{L}\left(\mathcal{S}_{\mathcal{P}}\right)$.

Proof. By Corollary 3, there exists $L \in \mathscr{G}\left(\mathbb{P}^{2}(\mathbb{K})\right)$ such that $\mathcal{M}={ }^{L} \mathcal{S}_{\mathcal{P}}$. Now, by Lemma $18, \mathcal{L}\left(\mathcal{S}_{\mathcal{P}}\right)=$ $\mathcal{L}(\mathcal{M})$. Taking into account that $\left\{m_{1}, m_{2}, m_{3}\right\}$ are linearly independent, we get the result.

The previous results show that the solution to our problem lies in $\mathcal{L}\left(\mathcal{S}_{\mathcal{P}}\right)$. However, knowing $\mathcal{L}\left(\mathcal{S}_{\mathcal{P}}\right)$ implies knowing $\mathcal{S}_{\mathcal{P}}$, which is essentially our goal. In the following, we see how 
to achieve $\mathcal{L}\left(\mathcal{S}_{\mathcal{P}}\right)$ by simply knowing $\mathscr{B}\left(\mathcal{S}_{\mathcal{P}}\right)$ and the base point multiplicities; note that, under the hypotheses of this section, this information is given by $\mathcal{P}$.

Definition 7. Let $\mathcal{M}$ be a birational transformation of $\mathbb{P}^{2}(\mathbb{K})$. We define the linear system of base points of $\mathcal{M}$, and we denote it by $\mathscr{L}(\mathcal{M})$, as the linear system of curves, of degree $\operatorname{deg}(\mathcal{M})$,

$$
\mathscr{L}(\mathcal{M})=\left\{f \in \mathscr{V}_{\operatorname{deg}(\mathcal{M})} \mid \operatorname{mult}(A, \mathscr{C}(f)) \geq \sqrt{\operatorname{mult}(A, \mathscr{B}(\mathcal{M}))} \forall A \in \mathscr{B}(\mathcal{M})\right\}
$$

Observe that $\mathscr{L}(\mathcal{M})$ is the $\operatorname{deg}(\mathcal{M})$-linear system associated to the effective divisor

$$
\sum_{A \in \mathscr{B}(\mathcal{M})} \sqrt{\operatorname{mult}(A, \mathscr{B}(\mathcal{M}))} \cdot A
$$

Remark 6. We observe that if $\mathcal{M}$ satisfies Condition 1, in particular $\mathcal{S}_{\mathcal{P}}$, then $\mathscr{L}(\mathcal{M})$ is the $\operatorname{deg}\left(\mathcal{S}_{\mathcal{P}}\right)$-degree linear system generated by the effective divisor

$$
\sum_{A \in \mathscr{B}(\mathcal{P})} \sqrt{\operatorname{mult}(A, \mathscr{B}(\mathcal{P}))} \cdot A .
$$

The following lemma is a direct consequence of the definition above. In Section 4.1, we have mentioned that we will work with equivalence classes. The next lemma states that the $\operatorname{deg}\left(\mathcal{S}_{\mathcal{P}}\right)$-degree linear system generated by the effective divisor is invariant within the equivalence class, and hence we may take whatever representant for our computations.

Lemma 19. Let $\mathcal{M}$ be a birational transformation of $\mathbb{P}^{2}(\mathbb{K})$. If $L \in \mathscr{G}\left(\mathbb{P}^{2}(\mathbb{K})\right)$ then $\mathcal{L}(\mathcal{M})=\mathcal{L}\left({ }^{L} \mathcal{M}\right)$ and $\mathscr{L}(\mathcal{M})=\mathscr{L}\left({ }^{L} \mathcal{M}\right)$

The next lemma relates the $\mathcal{L}(\mathcal{M})$ and $\mathscr{L}(\mathcal{M})$.

Lemma 20. If $\mathcal{M}$ is a transversal birational map of $\mathbb{P}^{2}(\mathbb{K})$, then $\mathcal{L}(\mathcal{M}) \subset \mathscr{L}(\mathcal{M})$.

Proof. Let $\mathcal{M}=\left(m_{1}: m_{2}: m_{3}\right)$, let $f \in \mathcal{L}(\mathcal{M})$ and $A \in \mathscr{B}(\mathcal{M})$. Then, $\operatorname{deg}(f)=\operatorname{deg}(\mathcal{M})$. On the other hand

$$
\begin{aligned}
\operatorname{mult}(A, \mathscr{C}(f)) & \geq \min \left\{\operatorname{mult}\left(A, \mathscr{C}\left(m_{i}\right)\right) \mid i \in\{1,2,3\}\right\} \\
& =\operatorname{mult}\left(A, \mathscr{C}\left(V_{1}\right)\right) \\
& =\sqrt{\operatorname{mult}(A, \mathscr{B}(\mathcal{M}))}
\end{aligned}
$$

$(\mathcal{M}$ is transversal).

Therefore, $f \in \mathscr{L}(\mathcal{M})$.

Lemma 21. If $\mathcal{M}$ is a transversal birational map of $\mathbb{P}^{2}(\mathbb{K})$, then $\operatorname{dim}(\mathscr{L}(\mathcal{M}))=2$.

Proof. Let $\mathcal{M}=\left(m_{1}: m_{2}: m_{3}\right)$. By Lemmas 17 and 20 , we have that $\operatorname{dim}(\mathscr{L}(\mathcal{M})) \geq 2$. Let us assume that $\operatorname{dim}(\mathscr{L}(\mathcal{M}))=k>2$ and let $\left\{n_{1}, \ldots, n_{k+1}\right\}$ be a basis of $\mathscr{L}(\mathcal{M})$ where $n_{1}=m_{1}, n_{2}=m_{2}, n_{3}=$ $m_{3}$. Then

$$
\mathscr{L}(\mathcal{M})=\left\{\lambda_{1} n_{1}+\cdots+\lambda_{k+1} n_{k+1} \mid\left(\lambda_{1}: \cdots: \lambda_{k+1}\right) \in \mathbb{P}^{k+1}(\mathbb{K})\right\} .
$$

Now, we take three points in $\mathbb{P}^{2}(\mathbb{K})$ that will be crucial later. For their construction, we first consider an open Zariski subset $\Sigma \subset \mathbb{P}^{2}(\mathbb{K})$ where $\mathcal{M}: \Sigma \rightarrow \mathcal{M}(\Sigma) \subset \mathbb{P}^{2}(\mathbb{K})$ is a bijective map. Then, $\mathcal{M}(\Sigma)$ is a constructible set of $\mathbb{P}^{2}(\mathbb{K})$ (see e.g., Theorem 3.16 in [22]). Thus, $\mathbb{P}^{2}(\mathbb{K}) \backslash \mathcal{M}(\Sigma)$ can only contain finitely many lines. On the other hand, we consider the open subset $\Omega_{2} \subset \mathscr{G}\left(\mathbb{P}^{2}(\mathbb{K})\right)$ introduced in Lemma 4 , and we take $L=\left(L_{1}: L_{2}: L_{3}\right) \in \Omega_{2}$ such that a non-empty open subset 
of $\mathscr{C}\left(L_{1}\right)$ is included in $\mathcal{M}(\Sigma)$. We take three points $B_{1}, B_{2}, B_{3} \in \Sigma$ (this, in particular, implies that $\left.B_{1}, B_{2}, B_{3} \notin \mathscr{B}(\mathcal{M})\right)$ such that:

1. $\mathcal{M}\left(B_{1}\right) \neq \mathcal{M}\left(B_{2}\right)$

2. $\mathcal{M}\left(B_{1}\right), \mathcal{M}\left(B_{2}\right) \in \mathscr{C}\left(L_{1}\right)$,

3. $\mathcal{M}\left(B_{3}\right) \notin \mathscr{C}\left(L_{1}\right)$; note that $\mathcal{M}\left(B_{1}\right), \mathcal{M}\left(B_{2}\right), \mathcal{M}\left(B_{3}\right)$ are not on a line

Since $\mathcal{M}\left(B_{1}\right), \mathcal{M}\left(B_{2}\right), \mathcal{M}\left(B_{3}\right)$ are not collinear, the system

$$
\left\{\sum_{i=1}^{k+1} \lambda_{i} n_{i}\left(B_{j}\right)=0\right\}_{j \in\{1,2,3\}}
$$

has solution. Let $\left(b_{1}: \cdots: b_{k+1}\right)$ be a solution. Then, we consider the polynomials (say that $\left.L_{1}:=a_{1} t_{1}+a_{2} t_{2}+a_{3} t_{3}\right)$

$$
f(\bar{t}):=L_{1}(\mathcal{M})=a_{1} m_{1}+a_{2} m_{2}+a_{3} m_{3}, g(\bar{t}):=b_{1} n_{1}+\cdots+b_{k+1} n_{k+1} .
$$

We have that $\mathscr{C}(f)$ is irreducible because $L \in \Omega_{2}$. Moreover, $\operatorname{deg}(\mathscr{C}(f))=\operatorname{deg}(\mathscr{C}(g))$. In addition, $\mathscr{C}(f) \neq \mathscr{C}(g)$ : indeed, $B_{3} \in \mathscr{C}(g)$ and $B_{3} \notin \mathscr{C}(f)$ because otherwise

$$
\left(\begin{array}{lll}
m_{1}\left(B_{1}\right) & m_{2}\left(B_{1}\right) & m_{3}\left(B_{1}\right) \\
m_{1}\left(B_{2}\right) & m_{2}\left(B_{2}\right) & m_{3}\left(B_{2}\right) \\
m_{1}\left(B_{3}\right) & m_{2}\left(B_{3}\right) & m_{3}\left(B_{3}\right)
\end{array}\right)\left(\begin{array}{l}
a_{1} \\
a_{2} \\
a_{3}
\end{array}\right)=\left(\begin{array}{l}
0 \\
0 \\
0
\end{array}\right),
$$

and since $\mathcal{M}\left(B_{1}\right), \mathcal{M}\left(B_{2}\right), \mathcal{M}\left(B_{3}\right)$ are not collinear we get that $a_{1}=a_{2}=a_{3}=0$ that is a contradiction. Therefore, $\mathscr{C}(f)$ and $\mathscr{C}(g)$ do not share components. Thus, by Bézout's theorem the number of intersections of $\mathscr{C}(f)$ and $\mathscr{C}(g)$, properly counted, is $\operatorname{deg}(\mathcal{M})^{2}$. In addition, we oberve that $f \in$ $\mathcal{L}(\mathcal{M}) \subset \mathscr{L}(\mathcal{M})$ (see Lemma 20) and $g \in \mathscr{L}(\mathcal{M})$. Thus,

$$
\mathscr{B}(\mathcal{M}) \cup\left\{B_{1}, B_{2}\right\} \subset \mathscr{C}(f) \cap \mathscr{C}(g) .
$$

Therefore

$$
\begin{aligned}
\operatorname{deg}(\mathcal{M})^{2} & =\sum_{A \in \mathscr{C}(f) \cap \mathscr{C}(g)} \operatorname{mult}_{A}(\mathscr{C}(f), \mathscr{C}(g)) \\
& \geq \sum_{A \in \mathscr{B}(\mathcal{M})} \operatorname{mult}_{A}(\mathscr{C}(f), \mathscr{C}(g))+\sum_{A \in\left\{B_{1}, B_{2}\right\}} \operatorname{mult}_{A}(\mathscr{C}(f), \mathscr{C}(g)) \\
& \geq \sum_{A \in \mathscr{B}(\mathcal{M})} \operatorname{mult}_{A}(\mathscr{C}(f), \mathscr{C}(g))+2 \\
& \geq \sum_{A \in \mathscr{B}(\mathcal{M})} \operatorname{mult}(A, \mathscr{C}(f)) \operatorname{mult}(A, \mathscr{C}(g))+2 \\
& =\sum_{A \in \mathscr{B}(\mathcal{M})} \operatorname{mult}(A, \mathscr{B}(\mathcal{M}))+2 \\
& =\operatorname{mult}(\mathscr{B}(\mathcal{M}))+2
\end{aligned}
$$
(see Corollary 7 in [12]).

which is a contradiction.

Theorem 5. If $\mathcal{M}$ is a transversal birational map of $\mathbb{P}^{2}(\mathbb{K})$, then $\mathcal{L}(\mathcal{M})=\mathscr{L}(\mathcal{M})$.

Proof. By Lemma 20, $\mathcal{L}(\mathcal{M}) \subset \mathscr{L}(\mathcal{M})$. Thus, using Lemmas 17 and 21, we get the result. 


\section{Algorithm and Examples}

In this section, we use the previous results to derive an algorithm for determining polynomial parametrizations of rational surface, under the conditions stated in Section 4.1. For this purpose we first introduce an auxiliary algorithm for testing the transversality of parametrizations. In addition, we observe that we require to the input parametrization to be proper (i.e., birational). This can be checked for instance using the algorithms in [29].

Observe that Step 2 in Algorithm 1 provides a first direct filter to detect some non-transversal parametrizations, and Step 5 applies the characterization in Lemma 10. This justifies the next theorem.

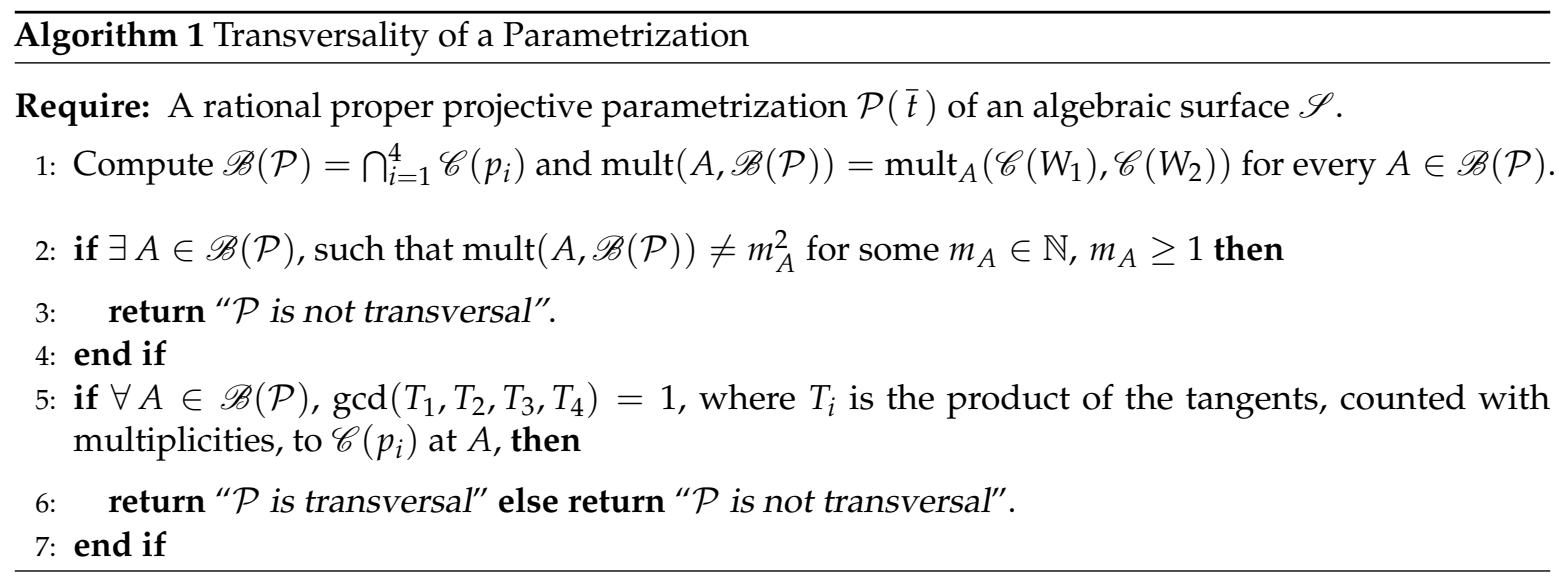

Theorem 6. Algorithm 1 is correct.

The following algorithm is the central algorithm of the paper.

Theorem 7. Algorithm 2 is correct.

Proof. For the correctness of the first steps (1-4) we refer to the preamble in Section 4 where the almost polynomial parametrizations are treated. For the rest of the steps, we use the notation introduced in Section 4 and we assume the hypotheses there, namely, $\mathcal{Q}\left(\mathcal{S}_{\mathcal{P}}\right)=\mathcal{P}$ and $\mathscr{B}(\mathcal{Q})=\varnothing$. Since $\mathcal{P}$ is transversal, by Theorem 1, we have that $\mathcal{S}_{\mathcal{P}}$ is transversal. Now, by Theorem $5, \mathscr{L}=\mathcal{L}\left(\mathcal{S}_{\mathcal{P}}\right)$. In this situation, by Theorem $4, \bar{S}={ }^{L} \mathcal{S}_{\mathcal{P}}$ for some $L \in \mathscr{G}\left(\mathbb{P}^{2}(\mathbb{K})\right)$. Therefore, $\mathcal{P}(\overline{\mathcal{R}})$ has to be almost polynomial, and hence the last step generates a polynomial parametrization. If the fourth component of $\mathcal{Q}$, namely $q_{4}$ is not the power of a linear form, then $\mathscr{B}(\mathcal{Q}) \neq \varnothing$.

Remark 7. Let us comment some consequences and computational aspects involved in the execution of the previous algorithms.

1. We observe that if Algorithm 2 returns a parametrization, then it is polynomial and its base locus is empty.

2. In order to check the properness of $\mathcal{P}$, one may apply, for instance, the results in [29] and, for determining $\operatorname{deg}(\mathscr{S})$, one may apply, for instance, the results in $[24,25,30]$. For the computation of $\overline{\mathcal{R}}$ one may apply well known elimination techniques as resultants or Gröbner basis; see e.g., [31].

3. In different steps of both algorithms one need to deal with the base points. Since the base locus is zero-dimensional, one may consider a decomposition of its elements in families of conjugate points, so that all further step can be performed exactly by introducing algebraic extensions of the ground field. For further details on how to deal with conjugate points we refer to Section 3.3 (Chapter 3) in [23]

We finish this section illustrating the algorithm with some examples. The first two examples provide polynomial parametrizations, while in the third the algorithm detects that the input parametrization, although proper, is not transversal. 


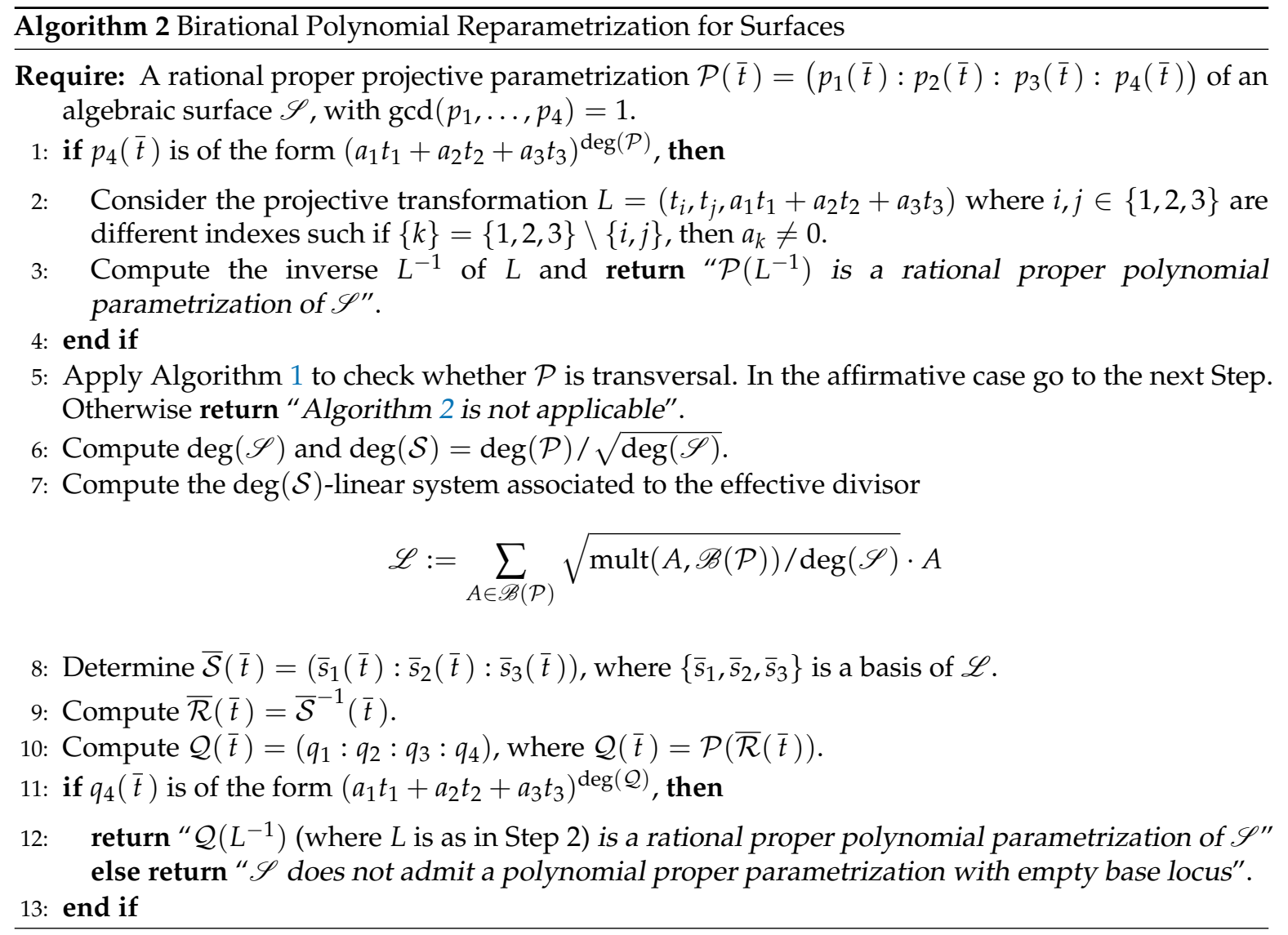

Example 1. Let $\mathcal{P}(\bar{t})=\left(p_{1}(\bar{t}): p_{2}(\bar{t}): p_{3}(\bar{t}): p_{4}(\bar{t})\right)$ be a rational parametrization of an algebraic surface $\mathscr{S}$, where

$$
\begin{aligned}
p_{1}= & -6 t_{3}^{4} t_{1} t_{2}+6 t_{3}^{2} t_{2}^{2} t_{1}^{2}-t_{3} t_{2} t_{1}^{4}-2 t_{3} t_{2}^{3} t_{1}^{2}+5 t_{3}^{3} t_{1}^{2} t_{2}+3 t_{3}^{3} t_{1} t_{2}^{2}-t_{2}^{6}+3 t_{3}^{2} t_{1}^{4}+3 t_{3}^{2} t_{2}^{4}- \\
& t_{3} t_{2}^{5}-3 t_{1}^{4} t_{2}^{2}-3 t_{1}^{2} t_{2}^{4}+t_{3}^{3} t_{1}^{3}-6 t_{3}^{4} t_{1}^{2}+3 t_{3}^{5} t_{1}+3 t_{3}^{3} t_{2}^{3}-6 t_{3}^{4} t_{2}^{2}+2 t_{3}^{5} t_{2}-t_{1}^{6} . \\
p_{2}= & -\left(t_{1}-t_{3}\right) t_{3}\left(t_{2}^{2}+t_{1}^{2}-t_{1} t_{3}\right)\left(t_{2}^{2}+t_{1}^{2}-2 t_{3}^{2}+t_{1} t_{3}\right) . \\
p_{3}= & t_{3}^{2} t_{2}^{3} t_{1}+t_{3}^{2} t_{1}^{3} t_{2}-3 t_{3}^{4} t_{1} t_{2}+39 t_{3}^{2} t_{2}^{2} t_{1}^{2}-8 t_{3} t_{2}^{2} t_{1}^{3}-4 t_{3} t_{1} t_{2}^{4}-4 t_{3} t_{2} t_{1}^{4}-8 t_{3} t_{2}^{3} t_{1}^{2}+8 t_{3}^{3} t_{1}^{2} t_{2} \\
& +6 t_{3}^{3} t_{1} t_{2}^{2}+6 t_{3}^{6}-5 t_{2}^{6}-4 t_{3} t_{1}^{5}+20 t_{3}^{2} t_{1}^{4}+19 t_{3}^{2} t_{2}^{4}-4 t_{3} t_{2}^{5}-15 t_{1}^{4} t_{2}^{2}-15 t_{1}^{2} t_{2}^{4}+8 t_{3}^{3} t_{1}^{3} \\
& -29 t_{3}^{4} t_{1}^{2}+4 t_{3}^{5} t_{1}+7 t_{3}^{3} t_{2}^{3}-22 t_{3}^{4} t_{2}^{2}-2 t_{3}^{5} t_{2}-5 t_{1}^{6} . \\
p_{4}= & \left(t_{1}^{2}+t_{2}^{2}-t_{3}^{2}\right)^{3} .
\end{aligned}
$$

Applying the results in [29], one deduces that $\mathcal{P}$ is proper. We apply Algorithm 2 in order to compute a rational proper polynomial parametrization $\mathcal{Q}(\bar{t})$ of $\mathscr{S}$, without base points, if it exists. Clearly, $\mathcal{P}$ is not almost polynomial and hence steps 1-4 does not apply. In Step 5, we perform Algorithm 1. The base locus is (we denote by $\pm \imath$ the square roots of -1 )

$$
\mathscr{B}(\mathcal{P})=\bigcap_{i=1}^{4} \mathscr{C}\left(p_{i}\right)=\{(1: 0: 1),(1: \imath: 0),(1:-\imath: 0)\}
$$

Moreover, it holds that

$$
\operatorname{mult}(A, \mathscr{B}(\mathcal{P}))=\operatorname{mult}_{A}\left(\mathscr{C}\left(W_{1}\right), \mathscr{C}\left(W_{2}\right)\right)=9, \forall A \in \mathscr{B}(\mathcal{P} .)
$$


Therefore, for every $A \in \mathscr{B}(\mathcal{P})$ we have that $\operatorname{mult}(A, \mathscr{B}(\mathcal{P}))=m_{A}^{2}$ for some $m_{A} \in \mathbb{N}, m_{A} \geq 1$. Thus, the necessary condition in Algorithm 1 is fulfilled. In addition, one may also check that the gcd of the tangents is 1 , for each base point. As a consequence, we deduce that $\mathcal{P}$ is transversal.

In Step 6 of Algorithm 2, we get that $\operatorname{deg}(\mathscr{S})=9$ (see [30]). Now, using that

$$
\operatorname{deg}(\mathcal{S})=\operatorname{deg}(\mathcal{P}) / \sqrt{\operatorname{deg}(\mathscr{S})}=6 / 3=2,
$$

and that

$$
\operatorname{mult}(A, \mathscr{B}(\mathcal{S}))=\operatorname{mult}(A, \mathscr{B}(\mathcal{P})) / \operatorname{deg}(\mathscr{S})=9 / 9=1 \quad \text { for every } A \in \mathscr{B}(\mathcal{P}),
$$

we compute the 2-linear system associated to the effective divisor

$$
\sum_{A \in \mathscr{B}(\mathcal{P})} A .
$$

For this purpose, one considers a generic polynomial of degree 2 with undetermined coefficients (note that we have 6 undetermined coefficients). We impose the three conditions, i.e., $\{(1: 0: 1),(1: \pm \imath: 0)\}$ should be simple points, and we get

$$
\mathscr{L}:=\lambda_{1}\left(-9 t_{1}^{2}-9 t_{2}^{2}+9 t_{1} t_{3}+t_{2} t_{3}\right)+\lambda_{2}\left(-10 t_{1}^{2}-10 t_{2}^{2}+9 t_{1} t_{3}+t_{3}^{2}\right)+\lambda_{3}\left(t_{1}^{2}+t_{2}^{2}-t_{3}^{2}\right) .
$$

Let

$$
\overline{\mathcal{S}}(\bar{t})=\left(\bar{s}_{1}: \bar{s}_{2}: \bar{s}_{3}\right)=\left(-9 t_{1}^{2}-9 t_{2}^{2}+9 t_{1} t_{3}+t_{2} t_{3}:-10 t_{1}^{2}-10 t_{2}^{2}+9 t_{1} t_{3}+t_{3}^{2}: t_{1}^{2}+t_{2}^{2}-t_{3}^{2}\right),
$$

where $\left\{\bar{s}_{1}, \bar{s}_{2}, \bar{s}_{3}\right\}$ is a basis of $\mathscr{L}$ Next, we compute

$$
\overline{\mathcal{R}}(\bar{t})=\overline{\mathcal{S}}^{-1}(\bar{t})=\left(\bar{r}_{1}(\bar{t}): \bar{r}_{2}(\bar{t}): \bar{r}_{3}(\bar{t})\right)=
$$

where

$$
\begin{aligned}
& \bar{r}_{1}=81 t_{1}^{2}-162 t_{1} t_{2}-162 t_{1} t_{3}+71 t_{3}^{2}+151 t_{2} t_{3}+80 t_{2}^{2}, \\
& \bar{r}_{2}=-9\left(2 t_{2}+11 t_{3}\right)\left(t_{1}-t_{2}-t_{3}\right), \\
& \bar{r}_{3}=181 t_{3}^{2}+82 t_{2}^{2}+81 t_{1}^{2}-162 t_{1} t_{2}+182 t_{2} t_{3}-162 t_{1} t_{3} .
\end{aligned}
$$

In the last step, the algorithm returns

$$
\mathcal{Q}(\bar{t})=\mathcal{P}(\overline{\mathcal{R}}(\bar{t}))=\left(t_{1}^{3}+t_{2} t_{3}^{2}-t_{1} t_{3}^{2}-t_{3}^{3}: t_{2}\left(t_{2}-t_{3}\right)\left(t_{2}+t_{3}\right): t_{2}^{3}+t_{1} t_{2}^{2}+t_{3} t_{2} t_{1}-4 t_{1} t_{3}^{2}-5 t_{3}^{3}: t_{3}^{3}\right)
$$

that is a rational proper polynomial parametrization of $\mathscr{S}$ with empty base locus. Note that the affine polynomial parametrization is given as

$$
\left(t_{1}^{3}+t_{2}-t_{1}-1, t_{2}\left(t_{2}-1\right)\left(t_{2}+1\right), t_{2}^{3}+t_{1} t_{2}^{2}+t_{2} t_{1}-4 t_{1}-5\right) .
$$

Observe that in this example we have introduced $\pm \imath$. Nevertheless we could have considered conjugate points. More precisely, the base locus decomposes as

$$
\{(1: 0: 1)\} \cup\left\{(1: s: 0) \mid s^{2}+1=0\right\}
$$

Then, all remaining computations could have been carried out working in the field extension $\mathbb{Q}(\alpha)$ where $\alpha^{2}+1=0$. 
Example 2. Let $\mathcal{P}(\bar{t})=\left(p_{1}(\bar{t}): p_{2}(\bar{t}): p_{3}(\bar{t}): p_{4}(\bar{t})\right)$ be a rational parametrization of an algebraic surface $\mathscr{S}$, where

$$
\begin{aligned}
& p_{1}=\frac{2891876933101}{7056} t_{2}^{4} t_{3}^{2}-\frac{94253497}{42} t_{2}^{5} t_{3}+\frac{79182089}{24} t_{1}^{5} t_{3}-\frac{15185833}{35} t_{2}^{5} t_{1}+\frac{230745016769}{19600} t_{2}^{4} t_{1}^{2} \\
& -\frac{789948757}{280} t_{1}^{4} t_{2}^{2}-\frac{314171}{4} t_{1}^{5} t_{2}-\frac{3324893202046}{2205} t_{2}^{3} t_{3}^{2} t_{1}+\frac{17297334852139}{29400} t_{2}^{2} t_{3}^{2} t_{1}^{2} \\
& +\frac{835536822991}{5880} t_{2}^{4} t_{3} t_{1}-\frac{3567593339657}{14700} t_{2}^{3} t_{3} t_{1}^{2}+\frac{8869391921}{420} t_{1}^{4} t_{2} t_{3}+\frac{199437407}{140} t_{1}^{3} t_{2}^{3} \\
& +\frac{56021820649}{144} t_{1}^{4} t_{3}^{2}+\frac{925548000997}{630} t_{1}^{3} t_{2} t_{3}^{2} \\
& -\frac{35094007283}{210} t_{1}^{3} t_{2}^{2} t_{3}+\frac{28561}{4} t_{2}^{6}+\frac{3455881}{16} t_{1}^{6}, \\
& p_{2}=-\frac{1097019300247}{2352} t_{2}^{4} t_{3}^{2}-\frac{246980149}{56} t_{2}^{5} t_{3}-2485483 t_{1}^{5} t_{3}-\frac{32737835}{56} t_{2}^{5} t_{1}-\frac{35410335273}{3920} t_{2}^{4} t_{1}^{2} \\
& +\frac{321945}{4} t_{1}^{4} t_{2}^{2}+\frac{314171}{4} t_{1}^{5} t_{2}+\frac{287134716635}{168} t_{2}^{3} t_{3}^{2} t_{1}-\frac{52659146973}{80} t_{2}^{2} t_{3}^{2} t_{1}^{2}-\frac{35536353385}{294} t_{2}^{4} t_{3} t_{1} \\
& +\frac{60928171523}{280} t_{2}^{3} t_{3} t_{1}^{2}+\frac{52899535}{3} t_{1}^{4} t_{2} t_{3}-\frac{446331197}{140} t_{1}^{3} t_{2}^{3}-\frac{5296771655}{12} t_{1}^{4} t_{3}^{2}-\frac{49879553251}{30} t_{1}^{3} t_{2} t_{3}^{2} \\
& +\frac{23802911463}{140} t_{1}^{3} t_{2}^{2} t_{3}-\frac{257049}{16} t_{2}^{6} \\
& p_{3}=-\frac{2676488123101}{7056} t_{2}^{4} t_{3}^{2}+\frac{94253497}{42} t_{2}^{5} t_{3}-\frac{379182089}{24} t_{1}^{5} t_{3}+\frac{15185833}{35} t_{2}^{5} t_{1}-\frac{2195132566369}{19600} t_{2}^{4} t_{1}^{2} \\
& +\frac{797945837}{280} t_{1}^{4} t_{2}^{2}+\frac{314171}{4} t_{1}^{5} t_{2}+\frac{3079803152296}{2205} t_{2}^{3} t_{3}^{2} t_{1}-\frac{16059945270739}{29400} t_{2}^{2} t_{3}^{2} t_{1}^{2} \\
& -\frac{786351504991}{5880} t_{2}^{4} t_{3} t_{1}+\frac{3371173762457}{14700} t_{2}^{3} t_{3} t_{1}^{2}-\frac{9628594001}{420} t_{1}^{4} t_{2} t_{3}-\frac{163616167}{140} t_{1}^{3} t_{2}^{3} \\
& -\frac{51903261673}{144} t_{1}^{4} t_{3}^{2}-\frac{857765630677}{630} t_{1}^{3} t_{2} t_{3}^{2}+\frac{32679676343}{210} t_{1}^{3} t_{2}^{2} t_{3}-\frac{28561}{4} t_{2}^{6}-\frac{3455881}{16} t_{1}^{6} \text {, } \\
& p_{4}=\left(-5348 t_{1}^{2} t_{3}+5525 t_{2}^{2} t_{3}+169 t_{1}^{2} t_{2}+757 t_{1} t_{2}^{2}-10059 t_{1} t_{2} t_{3}\right)^{2} .
\end{aligned}
$$

Applying the results in [29], one deduces that $\mathcal{P}$ is proper. We apply Algorithm 2. Clearly, $\mathcal{P}$ is not almost polynomial and hence steps 1-4 does not apply. In Step 5, we perform Algorithm 1. The base locus is

$$
\mathscr{B}(\mathcal{P})=\{(0: 0: 1),(1: 2: 1),(5: 7: 1),(1 / 3:-1 / 7: 1),(-13: 7: 1)\} .
$$

Moreover, it holds that

$$
\operatorname{mult}(A, \mathscr{B}(\mathcal{P}))=4
$$

for every $A \in \mathscr{B}(\mathcal{P})$ except for $A=(0: 0: 1)$ that satisfies that $\operatorname{mult}(A, \mathscr{B}(\mathcal{P}))=16$. Thus, the necessary condition in Algorithm 1 is fulfilled. In addition, one may also check that the gcd of the tangents is 1, for each base point. As a consequence, we deduce that $\mathcal{P}$ is transversal. Now, using that

$$
\operatorname{deg}(\mathcal{S})=\operatorname{deg}(\mathcal{P}) / \sqrt{\operatorname{deg}(\mathscr{S})}=6 / 2=3,
$$

and that

$$
\operatorname{mult}(A, \mathscr{B}(\mathcal{S}))=\operatorname{mult}(A, \mathscr{B}(\mathcal{P})) / \operatorname{deg}(\mathscr{S})=1,
$$

for every $A \in \mathscr{B}(\mathcal{P})$ except for $A=(0: 0: 1)$ that satisfies that $\operatorname{mult}(A, \mathscr{B}(\mathcal{S}))=4$, we compute the 3-linear system associated to the effective divisor

$$
4(0: 0: 1)+(1: 2: 1)+(5: 7: 1)+(1 / 3:-1 / 7: 1)+(-13: 7: 1) .
$$

We get that $\mathscr{L}=\lambda_{1} \bar{s}_{1}+\lambda_{2} \bar{s}_{2}+\lambda_{3} \bar{s}_{3}$ where

$$
\begin{aligned}
& \bar{s}_{1}=\frac{203971}{12} t_{1}^{2} t_{3}-\frac{1463501}{84} t_{2}^{2} t_{3}+\frac{3373732}{105} t_{1} t_{2} t_{3}+\frac{1859}{4} t_{1}^{3}+\frac{169}{2} t_{2}^{3}-\frac{169}{2} t_{1}^{2} t_{2}-\frac{438913}{140} t_{1} t_{2}^{2}, \\
& \bar{s}_{2}=\frac{37443}{2} t_{1}^{2} t_{3}-\frac{538707}{28} t_{2}^{2} t_{3}+\frac{140997}{4} t_{1} t_{2} t_{3}-\frac{507}{4} t_{2}^{3}-507 t_{1}^{2} t_{2}-\frac{71637}{28} t_{1} t_{2}^{2} \\
& \bar{s}_{2}=\frac{26747}{2} t_{1}^{2} t_{3}-\frac{384007}{28} t_{2}^{2} t_{3}-338 t_{1}^{2} t_{2}-\frac{50441}{28} t_{1} t_{2}^{2}+\frac{100761}{4} t_{1} t_{2} t_{3}-\frac{507}{4} t_{2}^{3} .
\end{aligned}
$$

So, we take, for instance, $\overline{\mathcal{S}}(\bar{t})=\left(\bar{s}_{1}(\bar{t}): \bar{s}_{2}(\bar{t}): \bar{s}_{3}(\bar{t})\right)$ and we compute $\overline{\mathcal{R}}(\bar{t})=\overline{\mathcal{S}}^{-1}(\bar{t})=\left(\bar{r}_{1}(\bar{t})\right.$ : $\left.\bar{r}_{2}(\bar{t}): \bar{r}_{3}(\bar{t})\right)$ where 


$$
\begin{aligned}
\bar{r}_{1}= & \frac{1}{11}\left(-34331 t_{2}+7140 t_{1}+39091 t_{3}\right)\left(-1240370879 t_{2}^{2}+4693319730 t_{2} t_{3}\right. \\
& \left.-957816090 t_{1} t_{2}-4096303731 t_{3}^{2}+26989200 t_{1}^{2}+1272637170 t_{1} t_{3}\right), \\
\bar{r}_{2}= & -\frac{7}{3}\left(-5349 t_{3}+3821 t_{2}\right)\left(-1240370879 t_{2}^{2}+4693319730 t_{2} t_{3}-957816090 t_{1} t_{2}\right. \\
& \left.-4096303731 t_{3}^{2}+26989200 t_{1}^{2}+1272637170 t_{1} t_{3}\right), \\
\bar{r}_{3}= & 9122349600 t_{1}^{2} t_{3}-6081566400 t_{1}^{2} t_{2}+5962839694227 t_{3}^{3}-13840668860013 t_{2} t_{3}^{2} \\
& +10640657052993 t_{2}^{2} t_{3}-2711599696487 t_{2}^{3}+503701536030 t_{1} t_{2}^{2} \\
& -1409880894660 t_{1} t_{2} t_{3}+985048833510 t_{1} t_{3}^{2} .
\end{aligned}
$$

Finally, we obtain

$$
\mathcal{Q}(\bar{t})=\mathcal{P}(\overline{\mathcal{R}}(\bar{t}))=\left(t_{1}^{2}+t_{2}^{2}-t_{2} t_{3}:-t_{1} t_{2}-t_{2}^{2}+t_{1} t_{3}:-t_{1}^{2}+t_{3}^{2}-t_{2} t_{3}:\left(t_{2}-t_{3}\right)^{2}\right) .
$$

Since $q_{4}(\bar{t})=\left(t_{2}-t_{3}\right)^{2}$, the algorithm returns

$$
\mathcal{Q}\left(\left(t_{1}, t_{2}, t_{2}-t_{3}\right)^{-1}\right)=\left(t_{1}^{2}+t_{2} t_{3}:-t_{2}^{2}-t_{1} t_{3}:-t_{1}^{2}+t_{3}^{2}-t_{2} t_{3}: t_{3}^{2}\right)
$$

that is a rational proper polynomial parametrization of $\mathscr{S}$ with empty base locus. Note that the affine polynomial parametrization is given as

$$
\left(t_{1}^{2}+t_{2},-t_{2}^{2}-t_{1},-t_{1}^{2}+1-t_{2}\right)
$$

Example 3. Let $\mathcal{P}(\bar{t})=\left(p_{1}(\bar{t}): p_{2}(\bar{t}): p_{3}(\bar{t}): p_{4}(\bar{t})\right)$ be a rational parametrization of an algebraic surface $\mathscr{S}$, where

$$
\begin{aligned}
p_{1}= & \left(-14065142 t_{1}^{3} t_{3}+29410550 t_{2}^{3} t_{3}-29410550 t_{2} t_{1}^{2} t_{3}+14065142 t_{2}^{2} t_{1} t_{3}+27633480 t_{1}^{4}\right. \\
& \left.-46976541 t_{1} t_{2}^{3}+64760061 t_{1}^{3} t_{2}\right)^{2} \\
p_{2}= & 15452942581758441 / 7 t_{2} t_{1}^{6} t_{3}-317479084729363299 / 49 t_{2}^{6} t_{1} t_{3} \\
& -68267697305871459 / 7 t_{2}^{5} t_{1}^{2} t_{3}-18666824719928010 / 7 t_{2}^{5} t_{1} t_{3}^{2} \\
& +212684946864036627 / 49 t_{2}^{4} t_{1}^{2} t_{3}^{2}+37333649439856020 / 7 t_{2}^{3} t_{1}^{3} t_{3}^{2} \\
& -2927680573060371 t_{2}^{2} t_{1}^{5} t_{3}-18666824719928010 / 7 t_{2} t_{1}^{5} t_{3}^{2} \\
& +10954535298967494 / 7 t_{2}^{3} t_{1}^{5}+1789545850442280 t_{2}^{2} t_{1}^{6}-1587369926524977 t_{2} t_{1}^{7} \\
& -700212410256675 t_{1}^{6} t_{3}^{2}+1255537783884564 t_{1}^{7} t_{3}+69932525820304176 / 7 t_{2}^{7} t_{1} \\
& -202783295759585328 / 49 t_{2}^{6} t_{1}^{2}-3042203660729001 t_{2}^{5} t_{1}^{3}+48537853394156778 / 7 t_{2}^{7} t_{3} \\
& -123497677483306851 / 49 t_{2}^{6} t_{3}^{2}+399414081398977842 / 49 t_{2}^{4} t_{1}^{3} t_{3} \\
& -4193865500723721 t_{2}^{8}-987075578994849 t_{1}^{8}-217339297920270 t_{2}^{4} t_{1}^{4} \\
& -54876861278152701 / 49 t_{2}^{2} t_{1}^{4} t_{3}^{2}+4276901329956240 / 7 t_{2}^{3} t_{1}^{4} t_{3}, \\
& 3 / 7\left(24511557 t_{1}^{4}-64760061 t_{1}^{2} t_{2}^{2}+11755445 t_{2} t_{1}^{2} t_{3}+38554704 t_{1} t_{2}^{3}-1125425 t_{2}^{2} t_{1} t_{3}\right. \\
& \left.-11755445 t_{2}^{3} t_{3}+1125425 t_{1}^{3} t_{3}\right)\left(-151106809 t_{2}^{4}+97487778 t_{2}^{3} t_{3}+269939512 t_{1} t_{2}^{3}\right. \\
& \left.+59811570 t_{2}^{2} t_{1} t_{3}-151106809 t_{1}^{2} t_{2}^{2}-97487778 t_{2} t_{1}^{2} t_{3}+98258706 t_{1}^{4}-59811570 t_{1}^{3} t_{3}\right) \\
p_{3}= & \left(24511557 t_{1}^{4}-64760061 t_{1}^{2} t_{2}^{2}+11755445 t_{2} t_{1}^{2} t_{3}+38554704 t_{1} t_{2}^{3}-1125425 t_{2}^{2} t_{1} t_{3}\right. \\
& \left.-11755445 t_{2}^{3} t_{3}+1125425 t_{1}^{3} t_{3}\right)^{2} . \\
p_{3}=1 &
\end{aligned}
$$


Applying the results in [29], one gets that $\mathcal{P}$ is proper. However, when applying Algorithm 1, we get that

$$
\mathscr{B}(\mathcal{P})=\{(0: 0: 1),(1: 2: 1),(5: 7: 1),(1 / 3:-1 / 7: 1),(-13: 7: 1)\}
$$

and that $\operatorname{mult}(A, \mathscr{B}(\mathcal{P}))=4$ for every $A \in \mathscr{B}(\mathcal{P})$ except for $A=(0: 0: 1)$ where $\operatorname{mult}(A, \mathscr{B}(\mathcal{P}))=44$. Since $\operatorname{mult}(A, \mathscr{B}(\mathcal{P}))=44$, which is not the square of a natural number, the algorithm returns that $\mathcal{P}$ is not transversal. Thus, we can not apply Algorithm 2.

\section{Conclusions}

Some crucial difficulties in many applications, and algorithmic questions, dealing with surface parametrizations are, on one hand, the presence of base points and, on the other, the existence of non-constant denominators of the parametrizations. In this paper, we have seen how to provide a polynomial parametrization with empty base locus, and hence an algorithm to avoid the two complications mentioned above, if it is possible. For this purpose, we have had to introduce, and indeed impose, the notion of transversal base locus. This notion directly affects to the transversality of the tangents at the base points of the algebraic plane curves $V_{i}$ or $W_{i}$ (see (3) and (7)). This, somehow, implies that in general one may expect transversality in the input. In any case, we do deal here with the non-transversal case and we leave it as an open problem. We think that using the ideas, pointed out by J. Schicho in [32], on blowing up the base locus, one might transform the given problem (via a finite sequence of Cremone transformations and projective transformations) into the case of transversality.

Author Contributions: The authors contributed equally to this work and they worked together through the whole paper. Both authors have read and agreed to the published version of the manuscript.

Funding: This work has been partially supported by FEDER/Ministerio de Ciencia, Innovación y Universidades-Agencia Estatal de Investigación/MTM2017-88796-P (Symbolic Computation: new challenges in Algebra and Geometry together with its applications).

Acknowledgments: Authors belong to the Research Group ASYNACS (Ref. CT-CE2019/683).

Conflicts of Interest: The authors declare no conflict of interest.

\section{References}

1. Hoschek, J.; Lasser, D. Fundamentals of Computer Aided Geometric Design; A.K. Peters, Ltd.: Natick, MA, USA, 1993.

2. Sendra, J.R.; Sevilla, D. First Steps Towards Radical Parametrization of Algebraic Surfaces. Comput. Aided Geom. Des. 2013, 30, 374-388. [CrossRef]

3. Schicho, J. Rational Parametrization of Surfaces. J. Symb. Comput. 1998, 26, 1-9. [CrossRef]

4. Sendra, J.R.; Sevilla, D.; Villarino, C. Algebraic and algorithmic aspects of radical parametrizations. Comput. Aided Geom. Des. 2017, 55,1-14. [CrossRef]

5. Bizzarri, M.; Lávička, M.; Vršek, J. Hermite interpolation by piecewise polynomial surfaces with polynomial area element. Comput. Aided Geom. Des. 2017, 51, 30-47. [CrossRef]

6. Šir, Z.; Gravesen, J.; Juttler, B. Curves and surfaces represented by polynomial support functions. Theor. Comput. Sci. 2008, 392, 141-157. [CrossRef]

7. Sendra, J.R. Normal Parametrization of Algebraic Plane Curves. J. Symb. Comput. 2002, 33, 863-885. [CrossRef]

8. Pérez-Díaz, S.; Sendra, J.R.; Villarino, C. A First Approach Towards Normal Parametrizations of Algebraic Surfaces. Int. J. Algebra Comput. 2010, 20, 977-990. [CrossRef]

9. Sendra, J.R.; Sevilla, D.; Villarino, C. Some results on the surjectivity of surface parametrizations. In Lecture Notes in Computer Science 8942; Schicho, J., Weimann, M., Gutierrez, J., Eds.; Springer International Publishing: Cham, Switzerland, 2015; pp. 192-203.

10. Grasegger, G. Radical solutions of first order autonomous algebraic ordinary differential equations. In Proceedings of the 39th International Symposium on Symbolic and Algebraic Computation, Kobe, Japan, 23-25 July 2014; Nabeshima, K., Ed.; ACM Press: New York, NY, USA, 2014; pp. 217-223.

11. Ngô, L.X.C.; Winkler, F. Rational general solutions of first order non-autonomous parametrizable ODEs. J. Symb. Comput. 2010, 45, 1426-1441. 
12. Cox, D.A.; Pérez-Díaz, S.; Sendra, J.R. On the base point locus of surface parametrizations: Formulas and consequences. arXiv 2020, arXiv:2008.08009.

13. Pérez-Díaz, S.; Sendra, J.R. Behavior of the Fiber and the Base Points of Parametrizations under Projections. Math. Comput. Sci. 2013, 7, 167-184. [CrossRef]

14. Busé, L.; Cox, D.; D'Andrea, C. Implicitization of surfaces in $\mathbb{P}^{3}$ in the presence of base points. J. Algebra Appl. 2003, 2, 189-214 [CrossRef]

15. Cox, D.; Goldman, R.; Zhang, M. On the validity of implicitization by moving quadrics for rational surfaces with no base points. J. Symb. Comput. 2000, 29, 419-440. [CrossRef]

16. Sendra, J.R.; Sevilla, D.; Villarino, C. Covering of surfaces parametrized without projective base points. In Proceedings of the 39th International Symposium on Symbolic and Algebraic Computation, Kobe, Japan, 23-25 July 2014; ACM Press: New York, NY, USA, 2014; pp. 375-380.

17. Shen, L.Y.; Goldman, R. Strong $\mu$-Bases for Rational Tensor Product Surfaces and Extraneous Factors Associated to Bad Base Points and Anomalies at Infinity. J. Appl. Algebra Geom. 2017, 1, 328-351. [CrossRef]

18. Arrondo, E.; Sendra, J.; Sendra, J.R. Parametric generalized offsets to hypersurfaces. J. Symb. Comput. 1997, 23, 267-285. [CrossRef]

19. Sendra, J.R.; Peternell, M.; Sendra, J. Cissoid Constructions of Augmented Rational Ruled Surfaces. Comput. Aided Geom. Des. 2018, 60, 1-9. [CrossRef]

20. Sendra, J.; Sendra, J.R. An algebraic analysis of conchoids to algebraic curves. Appl. Algebra Eng. Commun. Comput 2008, 19, 413-428. [CrossRef]

21. Vršek, J. Lávička M. On convolutions of algebraic curves. J. Symb. Comput. 2010, 45, 657-676. [CrossRef]

22. Harris, J. Algebraic Geometry. A First Course; Springer Science and Business Media: New York, NY, USA, 1995.

23. Sendra, J.R.; Winkler, F.; Pérez-Díaz, S. Rational Algebraic Curves: A Computer Algebra Approach. In Algorithms and Computation in Mathematics; Springer: Berlin/Heilderbarg, Germany, 2007; Volume 22.

24. Pérez-Díaz, S.; Sendra, J.R. A Univariate Resultant Based Implicitization Algorithm for Surfaces. J. Symb. Comput. 2008, 43, 118-139. [CrossRef]

25. Pérez-Díaz, S.; Sendra, J.R. Partial Degree Formulae for Rational Algebraic Surfaces. In Proceedings of the International Symposium on Symbolic and Algebraic Computation (ISSAC), Beijing, China, 24-27 July 2005; ACM Press: New York, NY, USA, 2005; pp. 301-308.

26. Pérez-Díaz, S.; Sendra, J.R.; Schicho, J. Properness and Inversion of Rational Parametrizations of Surfaces. Appl. Algebra Eng. Commun. Comput. 2002, 13, 29-51. [CrossRef]

27. Miranda, R. Linear Systems of Plane Curves. Notices AMS 46 1999, 2, 192-201.

28. Walker, R.J. Algebraic Curves; Princeton University Press: Princeton, NJ, USA, 1950.

29. Pérez-Díaz, S.; Sendra, J.R. Computation of the Degree of Rational Surface Parametrizations. J. Pure Appl. Algebra 2004, 193, 99-121. [CrossRef]

30. Pérez-Díaz, S.; Sendra, J.R.; Villarino, C. Computing the Singularities of Rational Surfaces. Math. Comput. 2015, 84, 1991-2021. [CrossRef]

31. Schicho, J. Inversion of birational maps with Gröbner bases. In Gröbner Bases and Applications; Buchberger, B., Winkler, F., Eds.; London Mathematical Society Lecture Note Series 251; Cambridge University Press: Cambridge, UK, 1998; pp. 495-503.

32. Schicho, J. Simplification of Surface Parametrizations. In Proceedings of the International Symposium on Symbolic and Algebraic Computation, Lille, France, 7-10 July 2002; ACM Press: New York, NY, USA, 2002; pp. 229-237.

Publisher's Note: MDPI stays neutral with regard to jurisdictional claims in published maps and institutional affiliations.

(C) 2020 by the authors. Licensee MDPI, Basel, Switzerland. This article is an open access article distributed under the terms and conditions of the Creative Commons Attribution (CC BY) license (http:/ / creativecommons.org/licenses/by/4.0/). 